\title{
Polynomial approximation with doubling weights *
}

\author{
Kirill A. Kopotun ${ }^{\dagger}$
}

August 9, 2021

\begin{abstract}
A nonnegative function $w \in \mathbb{L}_{1}[-1,1]$ is called a doubling weight if there is a constant $L$ such that $w(2 I) \leq L w(I)$, for all intervals $I \subset[-1,1]$, where $2 I$ denotes the interval having the same center as $I$ and twice as large as $I$, and $w(I):=$ $\int_{I} w(u) d u$. In this paper, we establish direct and inverse results for weighted approximation by algebraic polynomials in the $\mathbb{L}_{p}, 0<p \leq \infty$, (quasi)norm weighted by $w_{n}:=\rho_{n}(x)^{-1} \int_{x-\rho_{n}(x)}^{x+\rho_{n}(x)} w(u) d u$, where $\rho_{n}(x):=n^{-1} \sqrt{1-x^{2}}+n^{-2}$ and $w$ is a doubling weight.

Among other things, we prove that, for a doubling weight $w, 0<p \leq \infty$, $r \in \mathbb{N}_{0}$, and $0<\alpha<r+1-1 / \lambda_{p}$, we have

$$
E_{n}(f)_{p, w_{n}}=O\left(n^{-\alpha}\right) \Longleftrightarrow \omega_{\varphi}^{r+1}\left(f, n^{-1}\right)_{p, w_{n}}=O\left(n^{-\alpha}\right)
$$

where $\lambda_{p}:=p$ if $0<p<\infty, \lambda_{p}:=1$ if $p=\infty,\|f\|_{p, w}:=\left(\int_{-1}^{1}|f(u)|^{p} w(u) d u\right)^{1 / p}$, $\|f\|_{\infty, w}:=\operatorname{ess}_{\sup _{u \in[-1,1]}}(|f(u)| w(u)), \omega_{\varphi}^{r}(f, t)_{p, w}:=\sup _{0<h \leq t}\left\|\Delta_{h \varphi(\cdot)}^{r}(f, \cdot)\right\|_{p, w}$, $E_{n}(f)_{p, w}:=\inf _{P_{n} \in \Pi_{n}}\left\|f-P_{n}\right\|_{p, w}$, and $\Pi_{n}$ is the set of all algebraic polynomials of degree $\leq n-1$.

We will also introduce classes of doubling weights $\mathcal{W}^{\delta, \gamma}$ with parameters $\delta, \gamma \geq 0$ that are used to describe the behavior of $w_{n}(x) / w_{m}(x)$ for $m \leq n$. It turns out that every class $\mathcal{W}^{\delta, \gamma}$ with $(\delta, \gamma) \in \Upsilon:=\left\{(\delta, \gamma) \in \mathbb{R}^{2} \mid \delta \geq 1, \gamma \geq 0, \delta+\gamma \geq 2\right\}$ contains all doubling weights $w$, and for each pair $(\delta, \gamma) \notin \Upsilon$, there is a doubling weight not in $\mathcal{W}^{\delta, \gamma}$. We will establish inverse theorems and equivalence results similar to $(*)$ for doubling weights from classes $\mathcal{W}^{\delta, \gamma}$. Using the fact that $1 \in \mathcal{W}^{0,0}$, we get the well known inverse results and equivalences of type $(*)$ for unweighted polynomial approximation as an immediate corollary.

Equivalence type results involving related $K$-functionals and realization type results (obtained as corollaries of our estimates) are also discussed.

Finally, we mention that $(*)$ closes a gap left in the paper by G. Mastroianni and V. Totik "Best Approximation and moduli of smoothness for doubling weights", J. Approx. Theory 110 (2001), 180-199, where (*) was established for $p=\infty$ and $\omega_{\varphi}^{r+2}$ instead of $\omega_{\varphi}^{r+1}$ (it was shown there that, in general, $(*)$ is not valid for $p=\infty$ if $\omega_{\varphi}^{r+1}$ is replaced by $\left.\omega_{\varphi}^{r}\right)$.
\end{abstract}

*AMS classification: 41A10, 41A17, 41A25, 41A27. Keywords and phrases: Weighted polynomial approximation, Doubling weights, Moduli of smoothness, Degree of approximation.

${ }^{\dagger}$ Department of Mathematics, University of Manitoba, Winnipeg, Manitoba, R3T 2N2, Canada (kopotunk@cc. umanitoba.ca). Supported by NSERC of Canada. 


\section{Introduction and main results}

As usual, $\mathbb{L}_{p}(I), 0<p \leq \infty$, denotes the set of all measurable on $I$ functions $f$ equipped with the (quasi)norm $\|f\|_{\mathbb{L}_{p}(I)}$, and $\|f\|_{p}:=\|f\|_{\mathbb{L}_{p}[-1,1]}$. For a (nonnegative) weight function $w$ and $I \subset[-1,1]$ denote

$$
\|f\|_{\mathbb{L}_{p}(I), w}:=\left(\int_{I}|f(u)|^{p} w(u) d u\right)^{1 / p} \quad \text { and } \quad\|f\|_{\mathbb{L}_{\infty}(I), w}:=\operatorname{ess} \sup _{u \in I}(|f(u)| w(u)) .
$$

We also let $\|f\|_{p, w}:=\|f\|_{\mathbb{L}_{p}[-1,1], w}$. Note that $\|f\|_{\mathbb{L}_{p}(I), w}=\left\|w^{1 / p} f\right\|_{\mathbb{L}_{p}(I)}$ if $0<p<\infty$, and $\|f\|_{\mathbb{L}_{\infty}(I), w}=\|w f\|_{\mathbb{L}_{\infty}(I)}$ if $p=\infty$. Assuming for convenience that $w$ is identically zero outside $[-1,1]$, we recall that $w$ is called a doubling weight if there is a constant $L$ (the so-called doubling constant of the weight $w$ ) such that

$$
\int_{2 I} w(u) d u \leq L \int_{I} w(u) d u
$$

for all intervals $I \subset[-1,1]$, where $2 I$ denotes the interval having the same center as $I$ and twice as large as $I$. We note that the class of doubling weights is quite large, for example, all generalized Jacobi weights are doubling. Also, they are closely related to Muckenhoupt's $A_{p}, 1 \leq p<\infty$, weights all of which are contained in the so-called $A_{\infty}$ class of weights that assign to a subset of an interval $I \subset[-1,1]$ a "fair" share of the weight of $I$. We refer the reader to [21, Chapter V] for details on $A_{p}$ and $A_{\infty}$ classes, their characterizations and properties, and to the series of papers [13 16] for detailed discussions of various properties of doubling weights.

Following [13 16], for a weight $w \in \mathbb{L}_{1}$, we set

$$
w_{n}(x):=\frac{1}{\rho_{n}(x)} \int_{x-\rho_{n}(x)}^{x+\rho_{n}(x)} w(u) d u
$$

where $\rho_{n}(x):=n^{-1} \sqrt{1-x^{2}}+n^{-2}$.

If $r \in \mathbb{N}$, the weighted modulus of smoothness is defined by

$$
\omega_{\varphi}^{r}(f, t)_{p, w}:=\sup _{0<h \leq t}\left\|\Delta_{h \varphi(\cdot)}^{r}(f, \cdot)\right\|_{p, w}
$$

where $\varphi(x):=\sqrt{1-x^{2}}$ and

$$
\Delta_{h}^{r}(f, x,[a, b]):= \begin{cases}\sum_{i=0}^{r}\left(\begin{array}{l}
r \\
i
\end{array}\right)(-1)^{r-i} f(x-r h / 2+i h), & \text { if } x \pm r h / 2 \in[a, b], \\
0, & \text { otherwise, }\end{cases}
$$

is the $r$ th symmetric difference, $\Delta_{h}^{r}(f, x):=\Delta_{h}^{r}(f, x,[-1,1])$.

Throughout this paper, we use the standard notation, i.e., $\mathbb{N}$ is the set of all positive integers, $\mathbb{N}_{0}:=\mathbb{N} \cup\{0\}, \mathbb{R}_{+}:=[0, \infty), \Pi_{n}$ is the set of all algebraic polynomials of degree $\leq n-1, c$ are positive constants that may be different even if they occur in the same line, $A \sim B$ means that $c A \leq B \leq c A$, for some constants $c$ that do not depend on the "important" variables (what's "important" is usually clear from the context). We also use the notation $c_{*}, c^{*}$ and $c_{i}\left(i \in \mathbb{N}_{0}\right)$ for constants that we need to refer to, but those 
stay fixed only inside the lemmas where they are introduced (to make this explicit, we use $c_{*}$, for example, in several statements, but none of these constants are assumed to be the same). Additionally, $E_{n}(f)_{p, w}:=\inf _{P_{n} \in \Pi_{n}}\left\|f-P_{n}\right\|_{p, w}$ is the rate of best weighted approximation with weight $w$ of $f$ by algebraic polynomials of degree $\leq n-1$.

The following theorem that motivated this work was proved in [13, Theorem 1.1].

Theorem A. Let $w$ be a doubling weight and $r \in \mathbb{N}$. Then there is a constant $c_{*}$ depending only on $r$ and the doubling constant of $w$ such that we have for any $f$

$$
E_{n}(f)_{\infty, w_{n}} \leq c_{*} \omega_{\varphi}^{r}(f, 1 / n)_{\infty, w_{n}} .
$$

Conversely,

$$
\omega_{\varphi}^{r+2}\left(f, n^{-1}\right)_{\infty, w_{n}} \leq \frac{c_{*}}{n^{r}} \sum_{k=1}^{n} k^{r-1} E_{k}(f)_{\infty, w_{k}} .
$$

It immediately follows from Theorem $\mathrm{A}$ that, for $0<\alpha<r$,

$$
E_{n}(f)_{\infty, w_{n}}=O\left(n^{-\alpha}\right) \Longleftrightarrow \omega_{\varphi}^{r+2}\left(f, n^{-1}\right)_{\infty, w_{n}}=O\left(n^{-\alpha}\right)
$$

and it was shown in [13, p. 183] that (1.1) is no longer true if $\omega_{\varphi}^{r+2}$ is replaced by $\omega_{\varphi}^{r}$ (in the case $r=1$ ). Among other things, we show in this paper that (1.1) holds if $\omega_{\varphi}^{r+2}$ is replaced by $\omega_{\varphi}^{r+1}$ (see Corollary 7.8 ).

In this paper, we prove direct and inverse theorems for all $0<p \leq \infty$. For example, we prove that, if $w$ is a doubling weight, $r \in \mathbb{N}, 0<p \leq \infty$ and $f \in \mathbb{L}_{p}[-1,1]$, then for every $n \geq r$,

$$
E_{n}(f)_{p, w_{n}} \leq c \omega_{\varphi}^{r}(f, 1 / n)_{p, w_{n}}
$$

Conversely,

$$
\omega_{\varphi}^{r}\left(f, n^{-1}\right)_{p, w_{n}} \leq \frac{c}{n^{r-1 / \lambda_{p}}} \sum_{k=1}^{n} k^{r-1-1 / \lambda_{p}} E_{k}(f)_{p, w_{k}}, \quad 1 \leq p \leq \infty,
$$

and

$$
\omega_{\varphi}^{r}\left(f, \vartheta n^{-1}\right)_{p, w_{n}} \leq \frac{c}{n^{r-1 / p}}\left(\sum_{k=1}^{n} k^{r p-2} E_{k}(f)_{p, w_{k}}^{p}\right)^{1 / p}, \quad 0<p<1,
$$

where $0<\vartheta \leq 1$ is some constant, $\lambda_{p}:=p$, if $p<\infty$, and $\lambda_{\infty}:=1$, if $p=\infty$.

This implies that, for any doubling weight $w, r \in \mathbb{N}, 0<p \leq \infty$ and $f \in \mathbb{L}_{p}[-1,1]$, for $0<\alpha<r-1 / \lambda_{p}$ we have

$$
E_{n}(f,[-1,1])_{p, w_{n}}=O\left(n^{-\alpha}\right) \Longleftrightarrow \omega_{\varphi}^{r}\left(f, n^{-1}\right)_{p, w_{n}}=O\left(n^{-\alpha}\right) .
$$

In fact, we prove the inverse theorems in a more general way. In Section 2, we introduce classes of doubling weights $\mathcal{W}^{\delta, \gamma}$ with parameters $\delta, \gamma \geq 0$ that are used to describe the behavior of $w_{n}(x) / w_{m}(x)$ for $m \leq n$. It turns out that every class $\mathcal{W}^{\delta, \gamma}$ with $(\delta, \gamma) \in \Upsilon=\left\{(\delta, \gamma) \in \mathbb{R}^{2} \mid \delta \geq 1, \gamma \geq 0, \delta+\gamma \geq 2\right\}$ contains all doubling weights $w$, and for each class $\mathcal{W}^{\delta, \gamma}$ with $(\delta, \gamma) \notin \Upsilon$, there is a doubling weight not in this class. We will establish inverse theorems for doubling weights from classes $\mathcal{W}^{\delta, \gamma}$ for all $\delta, \gamma \geq 0$. Since positive constants are doubling weights from the class $\mathcal{W}^{0,0}$, we get the well known inverse results for unweighted polynomial approximation as an immediate corollary. 
The paper is organized as follows. In Section 2, we discuss several properties of weights $w_{n}$ and introduce classes $\mathcal{W}^{\delta, \gamma}$. An auxiliary result on a polynomial partition of unity that is crucial in our proof of direct results is introduced in Section 3 . In Section 4, we approximate the weights $w_{n}^{1 / p}$ by polynomials from $\Pi_{n}$. Section 5 is devoted to proving Jackson type (i.e., direct) results on polynomial approximation with weights $w_{n}$. Markov-Bernstein type results are discussed in Section 6. A major part of this section is devoted to the case $0<p<1$ in preparation for inverse results for these $p$. The inverse theorems are proved in Section 7 and, in Section 8 , we discuss some results on the equivalence of the moduli $\omega_{\varphi}^{r}$ as well as the averaged moduli $\tilde{\omega}_{\varphi}^{r}$ and several $K$-functionals and Realization functionals with weights $w_{n}$.

It is also worth mentioning that it seems possible to get the Jackson-type results in the case $1 \leq p \leq \infty$ using the Jackson-Favard type inequalities proved in [15, 16] and equivalence of the moduli $\omega_{\varphi}^{r}$ with weights $w_{n}$ and related $K$-functionals that can be obtained following proofs in [7] (as was done in [13], see also [4]). However, we opted for a different approach in this paper that works in the case $0<p<1$ as well.

\section{Doubling weights and their subclasses}

In this section, we discuss several properties of $w_{n}$ that will be used in this paper. First, we note that it was proved in [14, Lemma 7.1] that the doubling condition is equivalent to

$$
w_{n}(x) \leq K(1+n|x-y|+n|\varphi(x)-\varphi(y)|)^{s} w_{n}(y), \quad n \in \mathbb{N}, \quad x, y \in[-1,1],
$$

with some positive constants $K$ and $s$.

It immediately follows from (2.1) (see also [13, (2.3)]) that

$$
|x-y| \leq M \rho_{n}(x) \quad \Longrightarrow \quad w_{n}(x) \sim w_{n}(y)
$$

with equivalence constants depending only on $M$ and the doubling constant of $w$.

We will now discuss the relations between $w_{n}$ and $w_{m}$ for different $n$ and $m$. First of all, it is evident that, for any $x \in[-1,1]$,

$$
\rho_{n}(x) \leq \rho_{m}(x) \leq(n / m)^{2} \rho_{n}(x) \text { if } m \leq n .
$$

Therefore, since $w$ is nonnegative we have

$$
w_{n}(x) \leq(n / m)^{2} w_{m}(x), \quad m \leq n .
$$

Also, taking into account that $w$ is doubling and using [14, Lemmas 7.1 and 2.1(vi)] we have, for $m \leq n$ and $M:=n / m$,

$$
w_{m}(x) \leq \rho_{n}(x)^{-1} \int_{x-M^{2} \rho_{n}(x)}^{x+M^{2} \rho_{n}(x)} w(u) d u \leq c w_{n}(x),
$$

for some constant $c$ that depends on $M$ and the doubling constant of $w$. Hence, in particular,

$$
w_{n}(x) \sim w_{m}(x), \quad \text { if } n \sim m .
$$


It is rather obvious that (2.3) cannot be improved uniformly for all $x \in[-1,1]$ (for all doubling weights $w$ ) in the sense that it is no longer valid if $(n / m)^{2}$ is replaced by $(n / m)^{2-\varepsilon}$, for any $\varepsilon>0$ (see also Lemma 2.1 below). At the same time, it is clear that $(n / m)^{2}$ in (2.3) can be replaced by $(n / m)$ for $x$ that are "far" from the endpoints of $[-1,1]$. The following simple lemma makes this observation more precise and turns out to be crucial in our proofs of the inverse theorems.

Lemma 2.1. Let $w$ be a doubling weight, $m, n \in \mathbb{N}$ be such that $m \leq n$, and let

$$
(\delta, \gamma) \in \Upsilon:=\left\{(\delta, \gamma) \in \mathbb{R}^{2} \mid \delta \geq 1, \gamma \geq 0, \delta+\gamma \geq 2\right\}
$$

Then

$$
\frac{w_{n}(x)}{w_{m}(x)} \leq\left(\frac{n}{m}\right)^{\delta}\left(1+\frac{1}{m \varphi(x)}\right)^{\gamma}, \quad-1 \leq x \leq 1 .
$$

Moreover, if $(\delta, \gamma) \notin \Upsilon$ then there are doubling weights $w$ for which (2.5) is not valid.

Proof. Clearly, (2.5) is satisfied if $m=n$, and so we assume that $m \leq n-1$. Since $\rho_{n}(x) \leq \rho_{m}(x)$, we conclude that

$$
\int_{x-\rho_{n}(x)}^{x+\rho_{n}(x)} w(u) d u \leq \int_{x-\rho_{m}(x)}^{x+\rho_{m}(x)} w(u) d u
$$

and hence

$$
\frac{w_{n}(x)}{w_{m}(x)} \leq \frac{\rho_{m}(x)}{\rho_{n}(x)}=\frac{n}{m} \cdot \frac{\varphi(x)+1 / m}{\varphi(x)+1 / n}
$$

Therefore, (2.5) will be proved if we show that

$$
L:=\frac{\varphi(x)+1 / m}{\varphi(x)+1 / n} \leq\left(\frac{n}{m}\right)^{\delta-1}\left(1+\frac{1}{m \varphi(x)}\right)^{\gamma}=: R
$$

Now, if $\varphi(x) \geq(n-m)^{-1}$, then

$$
\begin{aligned}
L & \leq 1+\frac{1}{m \varphi(x)}=\left(1+\frac{1}{m \varphi(x)}\right)^{\gamma}\left(1+\frac{1}{m \varphi(x)}\right)^{1-\gamma} \\
& \leq\left(1+\frac{1}{m \varphi(x)}\right)^{\gamma} \max \left\{1,(n / m)^{1-\gamma}\right\} \leq R .
\end{aligned}
$$

If $\varphi(x)<(n-m)^{-1}$, then

$$
L=1+\frac{1 / m-1 / n}{\varphi(x)+1 / n} \leq \frac{n}{m} \leq\left(\frac{n}{m}\right)^{\delta+\gamma-1} \leq R .
$$

We will now construct examples showing that $(2.5)$ is no longer valid if $(\delta, \gamma) \notin \Upsilon$.

Let $R_{\delta, \gamma}(x)$ denote the right-hand side of (2.5). Since $\lim _{x \rightarrow 1} R_{\delta, \gamma}(x)=0$ if $\gamma<0$, it is obvious that $\gamma$ has to be nonnegative for (2.5) to hold (for example, if $\gamma<0$, then $1 \notin \mathcal{W}^{\delta, \gamma}$ for any $\left.\delta\right)$.

Now, let $w^{c}(x)=|x-c|^{-\alpha}$ with $c \in[-1,1]$ and $0<\alpha<1$. It is not difficult to see that $w^{c}$ is doubling and $\left(w^{c}\right)_{n}(x) \sim \min \left\{|x-c|^{-\alpha}, \rho_{n}(c)^{-\alpha}\right\}$. Hence, if $m \leq n$, then

$$
\frac{\left(w^{1}\right)_{n}\left(1-n^{-2}\right)}{\left(w^{1}\right)_{m}\left(1-n^{-2}\right)} \sim\left(\frac{n}{m}\right)^{2 \alpha} \quad \text { and } \quad R_{\delta, \gamma}\left(1-n^{-2}\right) \sim\left(\frac{n}{m}\right)^{\delta+\gamma} \text {. }
$$


Hence, if $\delta+\gamma<2$, then (2.5) does not hold for the doubling weights $w^{1}$ with $\max \{(\delta+$ $\gamma) / 2,0\}<\alpha<1$. Also,

$$
\frac{\left(w^{0}\right)_{n}(0)}{\left(w^{0}\right)_{m}(0)} \sim\left(\frac{n}{m}\right)^{\alpha} \quad \text { and } \quad R_{\delta, \gamma}(0) \sim\left(\frac{n}{m}\right)^{\delta},
$$

and so, if $\delta<1$, then (2.5) does not hold for the doubling weights $w^{0}$ with $\max \{\delta, 0\}<$ $\alpha<1$.

Definition 2.2. Let $\delta, \gamma \geq 0$. We say that a doubling weight $w$ belongs to the class $\mathcal{W}_{\Lambda}^{\delta, \gamma}$ if, for all $m, n \in \mathbb{N}$ such that $m \leq n$ and all $x \in[-1,1]$,

$$
w_{n}(x) \varphi(x)^{\gamma} \leq \Lambda n^{\delta} m^{\gamma-\delta} \rho_{m}(x)^{\gamma} w_{m}(x),
$$

for some constant $\Lambda$ which may depend only on the weight $w$, parameters $\delta$ and $\gamma$, and is independent of $m, n$ and $x$. We also denote

$$
\mathcal{W}^{\delta, \gamma}:=\left\{w \mid w \in \mathcal{W}_{\Lambda}^{\delta, \gamma} \text { for some } \Lambda>0\right\}
$$

We remark that (2.6) with $\Lambda=1$ is equivalent to (2.5) which is the reason for Definition 2.2. Also, it is evident that $\mathcal{W}^{\delta_{1}, \gamma_{1}} \subset \mathcal{W}^{\delta_{2}, \gamma_{2}}$ if $\delta_{1} \leq \delta_{2}$ and $\gamma_{1} \leq \gamma_{2}$.

Remark 2.3. Lemma 2.1 implies that all doubling weights belong to the class $\mathcal{W}_{1}^{\delta, \gamma}$ if $(\delta, \gamma) \in \Upsilon$, where $\Upsilon$ is defined in (2.4). Moreover, for any pair $(\delta, \gamma) \notin \Upsilon$, there is a doubling weight $w$ such that $w \notin \mathcal{W}^{\delta, \gamma}$.

Of course, there are many doubling weights belonging to the classes $\mathcal{W}^{\delta, \gamma}$ with $(\delta, \gamma) \notin$ $\Upsilon$. For example, any nonzero constant weight belongs to $\mathcal{W}^{0,0}$. The weight $w^{*}(x)=$ $(1-x)^{-\alpha}, 0<\alpha<1$, belongs to $\mathcal{W}^{\delta, \gamma}$ for all $(\delta, \gamma) \in \mathbb{R}_{+}^{2}$ such that $\delta+\gamma \geq 2 \alpha$. The weight $w_{*}(x)=|x|^{-\alpha}, 0<\alpha<1$, belongs to $\mathcal{W}^{\delta, \gamma}$ for all $(\delta, \gamma) \in \mathbb{R}_{+}^{2}$ such that $\delta \geq \alpha$. Hence, the doubling weight $W(x):=|x|^{-\alpha}(1-x)^{-\beta}, 0<\alpha, \beta<1$, which is a combination of $w^{*}$ and $w_{*}$ belongs to $\mathcal{W}^{\delta, \gamma}$ for all $(\delta, \gamma) \in \mathbb{R}_{+}^{2}$ such that $\delta \geq \alpha$ and $\delta+\gamma \geq 2 \beta$.

Following [13, 14] we say that a weight $w$ satisfies the $A^{*}$ property if there is a constant $c^{*}$ such that, for all $I \subset[-1,1]$ and $x \in I$,

$$
w(x) \leq \frac{c^{*}}{|I|} \int_{I} w(u) d u .
$$

Then $w$ is doubling and (2.2) implies that $w_{m}(x) \sim w_{m}(u)$ if $|x-u| \leq M \rho_{m}(x)$. Hence, if $m \leq n$, taking into account that $\rho_{n}(x) \leq \rho_{m}(x)$ we conclude that $w_{m}(x) \sim w_{m}(u)$ for $|x-u| \leq \rho_{n}(x)$. Therefore, denoting $J_{m}(u):=\left[u-\rho_{m}(u), u+\rho_{m}(u) \cap[-1,1]\right.$, we have (see also [13, p. 189])

$$
\begin{aligned}
w_{n}(x) & =\frac{1}{\rho_{n}(x)} \int_{x-\rho_{n}(x)}^{x+\rho_{n}(x)} w(u) d u \\
& \leq \frac{1}{\rho_{n}(x)} \int_{x-\rho_{n}(x)}^{x+\rho_{n}(x)}\left(\frac{c^{*}}{\left|J_{m}(u)\right|} \int_{J_{m}(u)} w(v) d v\right) d u \\
& \leq \frac{1}{\rho_{n}(x)} \int_{x-\rho_{n}(x)}^{x+\rho_{n}(x)}\left(\frac{c^{*}}{\rho_{m}(u)} \int_{u-\rho_{m}(u)}^{u+\rho_{m}(u)} w(v) d v\right) d u \\
& =\frac{c^{*}}{\rho_{n}(x)} \int_{x-\rho_{n}(x)}^{x+\rho_{n}(x)} w_{m}(u) d u \sim \frac{1}{\rho_{n}(x)} \int_{x-\rho_{n}(x)}^{x+\rho_{n}(x)} w_{m}(x) d u \sim w_{m}(x) .
\end{aligned}
$$

Therefore, we can make the following assertion. 
Remark 2.4. Any weight $w$ that satisfies the $A^{*}$ property is in the class $\mathcal{W}_{\Lambda}^{0,0}$ with the constant $\Lambda$ that depends only on the doubling constant of $w$.

Finally, we will need the following technical lemma that will be quite useful in the proofs of direct results (note that $x_{i}, I_{i}$ and $\psi_{i}$ are defined at the beginning of Section 3 ).

Lemma 2.5. For a doubling weight $w, n \in \mathbb{N}$ and all $1 \leq i \leq n, x \in[-1,1]$ and $y \in I_{i}$, we have

$$
w_{n}(x) \leq c \psi_{i}(x)^{-s} w_{n}(y) \quad \text { and } \quad w_{n}(y) \leq c \psi_{i}(x)^{-s} w_{n}(x)
$$

where constants $c$ and parameter $s \geq 0$ depend only on the doubling constant of $w$.

Proof. Taking into account that, for $1 \leq i \leq n-1,\left|I_{i}\right| \sim \varphi\left(x_{i}\right) / n$ and $\psi_{i}(x)^{-1}=$ $1+\left|x-x_{i}\right| /\left|I_{i}\right| \sim 1+n\left|x-x_{i}\right| / \varphi\left(x_{i}\right)$, and using (2.1) we have

$$
\begin{aligned}
& \max \left\{w_{n}(x) / w_{n}\left(x_{i}\right), w_{n}\left(x_{i}\right) / w_{n}(x)\right\} \leq c\left(1+n\left|x-x_{i}\right|+n\left|\varphi(x)-\varphi\left(x_{i}\right)\right|\right)^{s} \\
& \leq c\left(1+n\left|x-x_{i}\right|+\frac{n\left|x^{2}-x_{i}^{2}\right|}{\varphi(x)+\varphi\left(x_{i}\right)}\right)^{s} \leq c\left(1+n\left|x-x_{i}\right|+\frac{2 n\left|x-x_{i}\right|}{\varphi\left(x_{i}\right)}\right)^{s} \\
& \leq c\left(1+\frac{3 n\left|x-x_{i}\right|}{\varphi\left(x_{i}\right)}\right)^{s} \leq c \psi_{i}(x)^{-s}, \quad 1 \leq i \leq n-1 .
\end{aligned}
$$

Therefore, observing that (2.2) implies that $w_{n}(u) \sim w_{n}(y)$, for $u, y \in I_{i}, 1 \leq i \leq n$, and $\psi_{n}(x) \sim \psi_{n-1}(x)$, we get (2.7).

\section{Partition of unity}

First, we recall the usual setup for polynomial approximation (see e.g. [20]). Let $\left(x_{i}\right)_{i=0}^{n}$ be the Chebyshev partition of $[-1,1]$, i.e., $x_{i}=\cos (i \pi / n), 0 \leq i \leq n, I_{i}:=\left[x_{i}, x_{i-1}\right]$, $1 \leq i \leq n$,

$$
\psi_{i}:=\psi_{i}(x):=\frac{\left|I_{i}\right|}{\left|x-x_{i}\right|+\left|I_{i}\right|}, \quad 1 \leq i \leq n
$$

Then, for $1 \leq i \leq n$,

$$
\mathfrak{t}_{i}(x):=\left(\frac{\cos 2 n \arccos x}{x-x_{i}^{0}}\right)^{2}+\left(\frac{\sin 2 n \arccos x}{x-\bar{x}_{i}}\right)^{2}
$$

is an algebraic polynomial of degree $4 n-2$, where $\bar{x}_{i}:=\cos (i \pi / n-\pi / 2 n), 1 \leq i \leq n$, $x_{i}^{0}:=\cos (i \pi / n-\pi / 4 n), 1 \leq i<n / 2$, and $x_{i}^{0}:=\cos (i \pi / n-3 \pi / 4 n), n / 2 \leq i \leq n$. The following properties of the Chebyshev partition will often be used:

$$
\left|I_{i}\right| \sim \rho_{n}(x), x \in I_{i}, 1 \leq i \leq n, \quad \text { and } \quad\left|I_{i}\right| \sim\left|I_{i+1}\right|, 1 \leq i \leq n-1 .
$$

It is also convenient to denote

$$
\chi_{i}(x):=\chi_{\left[x_{i}, 1\right]}(x)= \begin{cases}1, & \text { if } x_{i} \leq x \leq 1 \\ 0, & \text { otherwise }\end{cases}
$$


The crucial (obvious) property of polynomials $\mathfrak{t}_{i}, 1 \leq i \leq n$, is

$$
\min \left\{\left(x-x_{i}^{0}\right)^{-2},\left(x-\bar{x}_{i}\right)^{-2}\right\} \leq \mathfrak{t}_{i}(x) \leq \max \left\{\left(x-x_{i}^{0}\right)^{-2},\left(x-\bar{x}_{i}\right)^{-2}\right\},
$$

which implies

$$
\mathfrak{t}_{i}(x) \sim\left(\left|x-x_{i}\right|+\left|I_{i}\right|\right)^{-2}
$$

uniformly for $x \in[-1,1]$.

There exists an absolute (positive) constant $c_{*}$ such that, for $\mu, \varepsilon_{1}, \varepsilon_{2} \in \mathbb{N}_{0}$ satisfying $\mu \geq c_{*} \max \left\{\varepsilon_{1}, \varepsilon_{2}, 1\right\}$,

$$
T_{i}(x):=T_{i}\left(n, \mu, \varepsilon_{1}, \varepsilon_{2}\right)(x):=\lambda_{i} \int_{-1}^{x}\left(y-x_{i}\right)^{\varepsilon_{1}}\left(x_{i-1}-y\right)^{\varepsilon_{2}} \mathfrak{t}_{i}^{\mu}(y) d y
$$

is a polynomial of degree $(4 n-2) \mu+\varepsilon_{1}+\varepsilon_{2}+1$, where

$$
\lambda_{i}:=\left(\int_{-1}^{1}\left(y-x_{i}\right)^{\varepsilon_{1}}\left(x_{i-1}-y\right)^{\varepsilon_{2}} \mathfrak{t}_{i}^{\mu}(y) d y\right)^{-1} \sim\left|I_{i}\right|^{2 \mu-\varepsilon_{1}-\varepsilon_{2}-1},
$$

and so $T_{i}(1)=1$ (see e.g. [11, Proposition 2]).

A proof of the following lemma is the same as that of [10, Lemmas 6]. It is based on (3.1), (3.2), the observation that

$$
\begin{aligned}
& \left|T_{i}(x)-\chi_{i}(x)\right|=\left|\int_{-1}^{x} T_{i}^{\prime}(u) d u\right|, \quad x<x_{i}, \\
& \left|T_{i}(x)-\chi_{i}(x)\right|=\left|\int_{x}^{1} T_{i}^{\prime}(u) d u\right|, \quad x>x_{i},
\end{aligned}
$$

and the Dzyadyk inequality (see e.g. [8, Theorem 3, p. 262])

$$
\left\|\rho_{n}^{s+\nu} P_{n}^{(\nu)}\right\|_{\infty} \leq c(s, \nu)\left\|\rho_{n}^{s} P_{n}\right\|_{\infty}, \quad P_{n} \in \Pi_{n} \quad \text { and } \quad s \in \mathbb{R} .
$$

Lemma 3.1. Let $1 \leq i \leq n$, and let $\nu_{0}, \mu, \varepsilon_{1}, \varepsilon_{2} \in \mathbb{N}_{0}$ be such that $\mu \geq c_{*} \max \left\{\nu_{0}, \varepsilon_{1}, \varepsilon_{2}, 1\right\}$, where $c_{*}$ is some sufficiently large absolute (positive) constant. Then the polynomial $T_{i}=$ $T_{i}\left(n, \mu, \varepsilon_{1}, \varepsilon_{2}\right)$ of degree $\leq c(\mu) n$ satisfies the following inequalities for all $x \in[-1,1]$ :

$$
\left|T_{i}(x)-\chi_{i}(x)\right| \leq c \psi_{i}(x)^{\mu}
$$

and

$$
\left|T_{i}^{(\nu)}(x)\right| \leq c\left|I_{i}\right|^{-\nu} \psi_{i}(x)^{\mu}, \quad 0 \leq \nu \leq \nu_{0},
$$

where constants $c$ depend only on $\mu$.

We note that by choosing $\varepsilon_{1}, \varepsilon_{2}$ to be 0 or 1 we can make polynomials $T_{i}\left(n, \mu, \varepsilon_{1}, \varepsilon_{2}\right)$ lie either above $\chi_{i-1}$ or below $\chi_{i}$. Indeed, recalling that $T_{i}(-1)=0$ and $T_{i}(1)=1$, inequalities $T_{i}^{\prime}(n, \mu, 1,0)(x)\left(x-x_{i}\right) \geq 0$ and $T_{i}^{\prime}(n, \mu, 0,1)(x)\left(x_{i-1}-x\right) \geq 0$ immediately imply that, for all $1 \leq i \leq n$,

$$
T_{i}(n, \mu, 1,0)(x) \leq \chi_{i}(x) \quad \text { and } \quad T_{i}(n, \mu, 0,1)(x) \geq \chi_{i-1}(x), \quad x \in[-1,1] .
$$




\section{Polynomial approximation of $w_{n}^{1 / p}$ for $0<p<\infty$}

Theorem 4.1. Suppose that $w$ is a doubling weight. For every $0<p<\infty, n \in \mathbb{N}$ and $\nu_{0} \in \mathbb{N}$, there exists a polynomial $\mathcal{Q}_{n} \in \Pi_{n}$ such that, for all $x \in[-1,1]$,

$$
c w_{n}(x)^{1 / p} \leq Q_{n}(x) \leq c w_{n}(x)^{1 / p}
$$

and

$$
\left|\rho_{n}(x)^{\nu} Q_{n}^{(\nu)}(x)\right| \leq c w_{n}(x)^{1 / p}, \quad 1 \leq \nu \leq \nu_{0},
$$

where constants $c$ depend only on $\nu_{0}, p$ and the doubling constant of $w$.

Note that, in the case $\nu_{0}=1$, Theorem 4.1 was proved in [14, (7.34)-(7.36)].

Proof. Let $S_{n}(x)$ be a piecewise constant function such that

$$
S_{n}(x)=s_{i}:=\sup _{u \in I_{i}} w_{n}(u)^{1 / p}, \quad x \in I_{i}, \quad 1 \leq i \leq n .
$$

Note that (2.2) implies that $w_{n}(x)^{1 / p} \leq s_{i} \leq c w_{n}(x)^{1 / p}$, for all $x \in I_{i}, 1 \leq i \leq n$, and so

$$
w_{n}(x)^{1 / p} \leq S_{n}(x) \leq c w_{n}(x)^{1 / p}, \quad x \in[-1,1] .
$$

We observe that

$$
S_{n}(x):=s_{n}+\sum_{i=1}^{n-1}\left(s_{i}-s_{i+1}\right) \chi_{i}(x), \quad x \in[-1,1]
$$

and define

$$
\mathcal{Q}_{n}(x):=s_{n}+\sum_{i=1}^{n-1}\left(s_{i}-s_{i+1}\right) R_{i}(x),
$$

where, for each $1 \leq i \leq n-1$, the polynomial $R_{i}$ is defined as follows

$$
R_{i}(x):= \begin{cases}T_{i+1}(n, \mu, 0,1), & \text { if } s_{i}-s_{i+1} \geq 0 \\ T_{i}(n, \mu, 1,0), & \text { otherwise }\end{cases}
$$

where $\mu \in \mathbb{N}$ is sufficiently large (to be prescribed). Then (3.4) yields

$$
S_{n}(x) \leq Q_{n}(x), \quad x \in[-1,1]
$$

which implies the left-hand inequality in (4.1). Now, for each $x \in[-1,1]$, using (2.7) and the fact that $\psi_{i+1} \sim \psi_{i}, 1 \leq i \leq n-1$, we have

$$
\begin{aligned}
\left|Q_{n}(x)-S_{n}(x)\right| & \leq \sum_{i=1}^{n-1}\left|s_{i}-s_{i+1}\right| \cdot\left|R_{i}(x)-\chi_{i}(x)\right| \leq c \sum_{i=1}^{n-1} w_{n}\left(x_{i}\right)^{1 / p} \psi_{i}(x)^{\mu} \\
& \leq c w_{n}(x)^{1 / p} \sum_{i=1}^{n-1} \psi_{i}(x)^{\mu-s / p} \leq c w_{n}(x)^{1 / p}
\end{aligned}
$$


since $\sum_{i=1}^{n-1} \psi_{i}(x)^{\mu-s / p} \leq c$ if $\mu-s / p \geq 2$. Therefore,

$$
Q_{n}(x) \leq S_{n}(x)+c w_{n}(x)^{1 / p} \leq c w_{n}(x)^{1 / p},
$$

which is the right-hand inequality in (4.1).

Now, recalling that $\left|I_{i}\right| \sim \rho_{n}\left(x_{i}\right), 1 \leq i \leq n$, and using the inequality $\rho_{n}(x)^{2} \leq$ $4 \rho_{n}(y)\left(|x-y|+\rho_{n}(y)\right)$ as well as (2.7) we have, for all $1 \leq \nu \leq \nu_{0}$,

$$
\begin{aligned}
\left|\rho_{n}(x)^{\nu} Q_{n}^{(\nu)}(x)\right| & \leq \sum_{i=1}^{n-1} \rho_{n}(x)^{\nu}\left|s_{i}-s_{i+1}\right| \cdot\left|R_{i}^{(\nu)}(x)\right| \leq c \sum_{i=1}^{n-1} \rho_{n}(x)^{\nu} w_{n}\left(x_{i}\right)^{1 / p}\left|I_{i}\right|^{-\nu} \psi_{i}(x)^{\mu} \\
& \leq c w_{n}(x)^{1 / p} \sum_{i=1}^{n-1}\left[\rho_{n}\left(x_{i}\right)\left(\left|x-x_{i}\right|+\rho_{n}\left(x_{i}\right)\right)\right]^{\nu / 2}\left|I_{i}\right|^{-\nu} \psi_{i}(x)^{\mu-s / p} \\
& \leq c w_{n}(x)^{1 / p} \sum_{i=1}^{n-1} \psi_{i}(x)^{\mu-s / p-\nu / 2} \leq c w_{n}(x)^{1 / p}
\end{aligned}
$$

provided $\mu-s / p-\nu / 2 \geq 2$. Hence, we choose $\mu$ to be such that all conditions of Lemma 3.1 are satisfied, and also $\mu \geq \nu_{0} / 2+s / p+2$. Finally, we note that we actually constructed a polynomial $Q_{n}$ of degree $\leq c(\mu) n$ that satisfies inequalities (4.1) and (4.2). Since $w_{n}(x) \sim w_{m}(x)$ and $\rho_{n}(x) \sim \rho_{m}(x)$ if $n \sim m$, this completes the proof for $n \geq n_{0}$, for some $n_{0} \in \mathbb{N}$. For $1 \leq n \leq n_{0}$, the statement of the theorem follows from the case $n=1$ (by setting $Q_{1}(x):=w_{1}(0)^{1 / p}$, for example).

\section{Weighted polynomial approximation: Jackson type estimates}

\subsection{Auxiliary results}

First, we recall the well known Whitney's theorem (see e.g. [19, Theorem 7.1, p. 195]) that states that, if $0<p \leq \infty, f \in \mathbb{L}_{p}[a, b]$ and $r \in \mathbb{N}$, then

$$
E_{r}(f,[a, b])_{p}:=\inf _{P_{r} \in \Pi_{r}}\left\|f-P_{r}\right\|_{\mathbb{L}_{p}[a, b]} \leq c \omega_{r}(f,(b-a) / r,[a, b])_{p},
$$

where $\omega_{r}(f, t,[a, b])_{p}$ is the usual $r$ th modulus of smoothness in the $\mathbb{L}_{p}$ (quasi)norm.

We also define the averaged weighted modulus by

$$
\begin{aligned}
\tilde{\omega}_{\varphi}^{r}(f, t)_{p, w} & :=\left(\frac{1}{t} \int_{0}^{t} \int_{-1}^{1} w(x)\left|\Delta_{h \varphi(x)}^{r}(f, x)\right|^{p} d x d h\right)^{1 / p} \\
& =\left(\frac{1}{t} \int_{0}^{t}\left\|\Delta_{h \varphi}^{r}(f)\right\|_{p, w}^{p} d h\right)^{1 / p}, \quad 0<p<\infty
\end{aligned}
$$

and for convenience denote $\tilde{\omega}_{\varphi}^{r}(f, t)_{\infty, w}:=\omega_{\varphi}^{r}(f, t)_{\infty, w}$.

Note that it is clear from the definition that

$$
\tilde{\omega}_{\varphi}^{r}(f, t)_{p, w} \leq \omega_{\varphi}^{r}(f, t)_{p, w}, \quad 0<p<\infty .
$$


Lemma 5.1. For a doubling weight $w, f \in \mathbb{L}_{p}[-1,1], 0<p<\infty, n, r \in \mathbb{N}$, and any $0<\theta<1$ the following holds

$$
\sum_{i=1}^{n} w_{n}\left(x_{i}\right) \omega_{r}\left(f,\left|J_{i}\right|, J_{i}\right)_{p}^{p} \leq c \tilde{\omega}_{\varphi}^{r}(f, \theta / n)_{p, w_{n}}^{p} \leq c \omega_{\varphi}^{r}(f, \theta / n)_{p, w_{n}}^{p}
$$

where, for every $i, I_{i} \subset J_{i} \subset[-1,1]$ and $\left|J_{i}\right| \leq c_{0}\left|I_{i}\right|$, and the constant $c$ depends only on $r, p, c_{0}, \theta$, and the doubling constant of $w$.

We remark that the reason for introducing $\theta$ is that we have NOT proved the estimate

$$
\omega_{\varphi}^{r}(f, \lambda / n)_{p, w_{n}} \leq c \omega_{\varphi}^{r}(f, 1 / n)_{p, w_{n}}, \quad p>0 .
$$

Proof. The proof of this lemma is rather standard and not different from that for unweighted moduli (see e.g. [2]). The main idea is the employment of the inequality (see [19, Lemma 7.2, p. 191])

$$
\omega_{r}(f, t,[a, b])_{p}^{p} \leq \frac{c}{t} \int_{0}^{t} \int_{a}^{b}\left|\Delta_{h}^{r}(f, x,[a, b])\right|^{p} d x d h, \quad 0<p<\infty .
$$

Note that if $J_{i} \supset I_{i}$ and $\left|J_{i}\right| \leq c_{0}\left|I_{i}\right|$, then there exists $m \in \mathbb{N}$ depending only on $c_{0}$ such that $J_{i}$ has nonempty intersection with at most $m$ intervals $I_{j}, 1 \leq j \leq n$. Since $\left|I_{i}\right| \sim\left|I_{i \pm 1}\right| \sim \rho_{n}\left(x_{i}\right)$, this implies that $\rho_{n}(x) \sim \rho_{n}(y) \sim\left|I_{i}\right|$ for all $x, y \in J_{i}$, and so $|x-y| \leq c \rho_{n}(x)$, for all $x, y \in J_{i}$.

Taking this into account and using (5.2) and (2.2) we have

$$
\begin{aligned}
w_{n}\left(x_{i}\right) \omega_{r}\left(f,\left|J_{i}\right|, J_{i}\right)_{p}^{p} & \leq c w_{n}\left(x_{i}\right) \omega_{r}\left(f, c^{*}\left|I_{i}\right|, J_{i}\right)_{p}^{p} \\
& \leq c\left|I_{i}\right|^{-1} \int_{0}^{c^{*}\left|I_{i}\right|} \int_{J_{i}} w_{n}\left(x_{i}\right)\left|\Delta_{h}^{r}\left(f, x, J_{i}\right)\right|^{p} d x d h \\
& \leq c \int_{J_{i}} \int_{0}^{c^{*}\left|I_{i}\right| / \varphi(x)} \frac{\varphi(x)}{\left|I_{i}\right|} w_{n}(x)\left|\Delta_{h \varphi(x)}^{r}\left(f, x, J_{i}\right)\right|^{p} d h d x,
\end{aligned}
$$

where $0<c^{*}<1$ is a constant that we will choose later.

Now, $\left|I_{i}\right| \sim \rho_{n}(x) \sim \varphi(x) / n$ for $x \in J_{i}, i \in J^{*}$, where

$$
J^{*}:=\left\{1 \leq i \leq n \mid J_{i} \cap\left(I_{1} \cup I_{n}\right)=\emptyset\right\},
$$

and so, for $i \in J^{*}$, taking into account that $c^{*} \leq \sqrt{c^{*}}$ (it is a red herring for now, but is needed because of the estimate for $i \notin J^{*}$ below), we have

$$
w_{n}\left(x_{i}\right) \omega_{r}\left(f,\left|J_{i}\right|, J_{i}\right)_{p}^{p} \leq c n \int_{J_{i}} \int_{0}^{c_{1} \sqrt{c^{*}} / n} w_{n}(x)\left|\Delta_{h \varphi(x)}^{r}\left(f, x, J_{i}\right)\right|^{p} d h d x .
$$

Suppose now that $i \notin J^{*}$. We recall that $\Delta_{h}^{r}\left(f, x, J_{i}\right)$ is defined to be 0 if $x \pm r h / 2 \notin J_{i}$ and, in particular, $\Delta_{h \varphi(x)}^{r}\left(f, x, J_{i}\right)=0$ if $1-|x|<r h \varphi(x) / 2$. Therefore, recalling that $\varphi(x) /\left|I_{i}\right| \leq c n \rho_{n}(x) /\left|I_{i}\right| \leq c n, x \in J_{i}$, for each fixed $x \in J_{i}$, we have

$$
\int_{0}^{c^{*}\left|I_{i}\right| / \varphi(x)} \frac{\varphi(x)}{\left|I_{i}\right|} w_{n}(x)\left|\Delta_{h \varphi(x)}^{r}\left(f, x, J_{i}\right)\right|^{p} d h \leq c n \int_{S} w_{n}(x)\left|\Delta_{h \varphi(x)}^{r}\left(f, x, J_{i}\right)\right|^{p} d h,
$$


where

$$
\begin{aligned}
S & :=\left\{h \mid 0<h \leq \min \left\{\frac{c^{*}\left|I_{i}\right|}{\varphi(x)}, \frac{2(1-|x|)}{r \varphi(x)}\right\}\right\} \\
& \subset\left\{h \mid 0<h \leq c_{2} \min \left\{\frac{c^{*}}{n^{2} \sqrt{1-|x|}}, \sqrt{1-|x|}\right\}\right\} \subset\left\{h \mid 0<h \leq c_{2} \sqrt{c^{*}} / n\right\} .
\end{aligned}
$$

Therefore, (5.3) is valid for $i \notin J^{*}$ as well (with $c_{2}$ instead of $c_{1}$ ). We now choose $c^{*}$ to be such that $\max \left\{c_{1}, c_{2}\right\} \sqrt{c^{*}}<\theta$. Then

$$
\begin{aligned}
\sum_{i=1}^{n} w_{n}\left(x_{i}\right) \omega_{r}\left(f,\left|J_{i}\right|, J_{i}\right)_{p}^{p} & \leq c n \sum_{i=1}^{n} \int_{J_{i}} \int_{0}^{\theta / n} w_{n}(x)\left|\Delta_{h \varphi(x)}^{r}\left(f, x, J_{i}\right)\right|^{p} d h d x \\
& \leq c n \sum_{i=1}^{n} \int_{I_{i}} \int_{0}^{\theta / n} w_{n}(x)\left|\Delta_{h \varphi(x)}^{r}(f, x)\right|^{p} d h d x \\
& \leq c n \int_{0}^{\theta / n} \int_{-1}^{1} w_{n}(x)\left|\Delta_{h \varphi(x)}^{r}(f, x)\right|^{p} d x d h \\
& \leq c \tilde{\omega}_{\varphi}^{r}(f, \theta / n)_{p, w_{n}}^{p},
\end{aligned}
$$

and the proof is complete.

An analog of Lemma 5.1 in the case $p=\infty$ is the following result.

Lemma 5.2. For a doubling weight $w, f \in \mathbb{L}_{\infty}[-1,1], n, r \in \mathbb{N}$, and any $0<\theta<1$ the following holds

$$
\sup _{1 \leq i \leq n} w_{n}\left(x_{i}\right) \omega_{r}\left(f,\left|J_{i}\right|, J_{i}\right)_{\infty} \leq c \omega_{\varphi}^{r}(f, \theta / n)_{\infty, w_{n}},
$$

where, for every $i, I_{i} \subset J_{i} \subset[-1,1]$ is such that $\left|J_{i}\right| \leq c_{0}\left|I_{i}\right|$, and the constant $c$ depends only on $r, c_{0}, \theta$, and the doubling constant of $w$.

Proof. Let $0<c^{*}<1 /(2 r)$ be a constant that we will prescribe later, and let $1 \leq i \leq n$, $x^{*} \in J_{i}$ and $h^{*} \in\left(0, c^{*}\left|I_{i}\right|\right]$ (note that $\left.h^{*}<1 / r\right)$ be such that

$$
\begin{aligned}
W & :=\sup _{1 \leq j \leq n} w_{n}\left(x_{j}\right) \omega_{r}\left(f,\left|J_{j}\right|, J_{j}\right)_{\infty}=w_{n}\left(x_{i}\right) \omega_{r}\left(f,\left|J_{i}\right|, J_{i}\right)_{\infty} \\
& \leq c w_{n}\left(x_{i}\right) \omega_{r}\left(f, c^{*}\left|I_{i}\right|, J_{i}\right)_{\infty} \leq c w_{n}\left(x_{i}\right)\left|\Delta_{h^{*}}^{r}\left(f, x^{*}\right)\right| .
\end{aligned}
$$

It was shown in the proof of Lemma 5.1, that $|x-y| \leq c \rho_{n}(x)$, for all $x, y \in J_{i}$, and so $w_{n}\left(x_{i}\right) \sim w_{n}\left(x^{*}\right)$.

Now, we set $h:=h^{*} / \varphi\left(x^{*}\right)$ and consider two cases: (i) $\varphi\left(x^{*}\right) \geq \theta /(2 n)$ and (ii) $\varphi\left(x^{*}\right)<\theta /(2 n)$. In the case (i), $\left|I_{i}\right| \sim \rho_{n}\left(x^{*}\right) \sim \varphi\left(x^{*}\right) / n$, and so $h \leq c c^{*} / n$ for some positive constant $c$, and we can choose $c^{*}$ so that $h \leq \theta / n$. In the case (ii), since $x^{*} \pm r h^{*} / 2 \in[-1,1]$, we conclude that $\varphi\left(x^{*}\right) \geq \sqrt{h^{*} / 2}$, and so $h^{*}<\theta^{2} /\left(2 n^{2}\right)$. Therefore, $h \leq \sqrt{2 h^{*}} \leq \theta / n$.

Hence, for some $0<h \leq \theta / n$,

$$
W \leq c w_{n}\left(x^{*}\right)\left|\Delta_{h \varphi\left(x^{*}\right)}^{r}\left(f, x^{*}\right)\right|,
$$

and so

$$
W \leq c \sup _{0<h \leq \theta / n} \sup _{x \in[-1,1]}\left|w_{n}(x) \Delta_{h \varphi(x)}^{r}(f, x)\right| \leq c \omega_{\varphi}^{r}(f, \theta / n)_{\infty, w_{n}}
$$




\section{$5.2 \quad$ Jackson type estimate}

Theorem 5.3. Let $w$ be a doubling weight, $r \in \mathbb{N}, 0<p \leq \infty$ and $f \in \mathbb{L}_{p}[-1,1]$. Then, for every $n \geq r$ and $0<\vartheta \leq 1$, there exists a polynomial $P_{n} \in \Pi_{n}$ such that

$$
\left\|f-P_{n}\right\|_{p, w_{n}} \leq c \tilde{\omega}_{\varphi}^{r}(f, \vartheta / n)_{p, w_{n}} \leq c \omega_{\varphi}^{r}(f, \vartheta / n)_{p, w_{n}}
$$

and

$$
\left\|\rho_{n}^{\nu} P_{n}^{(\nu)}\right\|_{p, w_{n}} \leq c \tilde{\omega}_{\varphi}^{r}(f, \vartheta / n)_{p, w_{n}} \leq c \omega_{\varphi}^{r}(f, \vartheta / n)_{p, w_{n}}, \quad r \leq \nu \leq \nu_{0}
$$

where constants $c$ depend only on $r, \nu_{0}, p, \vartheta$ and the doubling constant of $w$.

We remark that, in the case $p=\infty$, it is usually assumed that $f \in \mathbb{C}[-1,1]$ since, otherwise, $\omega_{\varphi}^{r}(f, 1 / n)_{p, w_{n}} \geq c>0, n \in \mathbb{N}$, and so the assumption that $f \in \mathbb{L}_{\infty}[-1,1]$ does not make this theorem more general.

Proof. We first assume that $0<p<\infty$. For $n \in \mathbb{N}$, let $\left(x_{i}\right)_{i=0}^{n}$ be the Chebyshev partition of $[-1,1]$, and let $p_{i} \in \Pi_{r}, 1 \leq i \leq n$, be a polynomial of near best approximation of $f$ on $J_{i}:=I_{i} \cup I_{i-1}$ (with $I_{0}:=\emptyset$ ) in the $\mathbb{L}_{p}$ (quasi)norm, i.e.,

$$
\left\|f-p_{i}\right\|_{\mathbb{L}_{p}\left(I_{i}\right)} \leq c E_{r}\left(f, J_{i}\right)_{p}
$$

We define $S_{n}$ to be a piecewise polynomial function such that $p_{i}=\left.S_{n}\right|_{I_{i}}, 1 \leq i \leq n$.

Then

$$
S_{n}(x)=p_{n}(x)+\sum_{i=1}^{n-1}\left[p_{i}(x)-p_{i+1}(x)\right] \chi_{i}(x) .
$$

Therefore, using (2.2), (5.1) and Lemma 5.1 we have

$$
\begin{aligned}
\left\|f-S_{n}\right\|_{p, w_{n}}^{p} & =\sum_{i=1}^{n} \int_{I_{i}} w_{n}(x)\left|f(x)-S_{n}(x)\right|^{p} d x \leq c \sum_{i=1}^{n} w_{n}\left(x_{i}\right) \int_{I_{i}}\left|f(x)-p_{i}(x)\right|^{p} d x \\
& \leq c \sum_{i=1}^{n} w_{n}\left(x_{i}\right) \omega_{r}\left(f,\left|I_{i}\right|, J_{i}\right)_{p}^{p} \leq c \tilde{\omega}_{\varphi}^{r}(f, \theta / n)_{p, w_{n}}^{p},
\end{aligned}
$$

where $0<\theta<1$ will be chosen later. We now define

$$
P_{n}(x):=p_{n}(x)+\sum_{i=1}^{n-1}\left[p_{i}(x)-p_{i+1}(x)\right] T_{i}(x),
$$

where $T_{i}=T_{1}\left(n, \mu, \varepsilon_{1}, \varepsilon_{2}\right)$ are the polynomials from Lemma 3.1 (note that the choice of $\varepsilon_{1}$ and $\varepsilon_{2}$ is not important; for example, we can set $\varepsilon_{1}=\varepsilon_{2}=0$ ) with a sufficiently large $\mu$ (we will prescribe it later so that all restrictions below are satisfied).

Lemma 3.1 now implies

$$
\begin{aligned}
\left\|S_{n}-P_{n}\right\|_{p, w_{n}}^{p} & \leq \int_{-1}^{1} w_{n}(x)\left[\sum_{i=1}^{n-1}\left|p_{i}(x)-p_{i+1}(x)\right| \cdot\left|\chi_{i}(x)-T_{i}(x)\right|\right]^{p} d x \\
& \leq c \int_{-1}^{1} w_{n}(x)\left[\sum_{i=1}^{n-1}\left\|p_{i}-p_{i+1}\right\|_{\infty} \psi_{i}(x)^{\mu}\right]^{p} d x
\end{aligned}
$$


Now, using the Lagrange interpolation formula and [3, Theorem 4.2.7] we have, for all $p \in \Pi_{r}$ and $0 \leq l \leq r-1$,

$$
\left\|p^{(l)}\right\|_{\infty} \leq c \psi_{i}^{-r+l+1}\left\|p^{(l)}\right\|_{\mathbb{C}\left(I_{i}\right)} \leq c \psi_{i}^{-r+l+1}\left|I_{i}\right|^{-l-1 / p}\|p\|_{\mathbb{L}_{p}\left(I_{i}\right)},
$$

and hence

$$
\left\|S_{n}-P_{n}\right\|_{p, w_{n}}^{p} \leq c \int_{-1}^{1} w_{n}(x)\left[\sum_{i=1}^{n-1}\left\|p_{i}-p_{i+1}\right\|_{\mathbb{L}_{p}\left(I_{i}\right)}\left|I_{i}\right|^{-1 / p} \psi_{i}(x)^{\mu-r+1}\right]^{p} d x .
$$

Now, if $1 \leq p<\infty$, since $\sum_{i=1}^{n-1} \psi_{i}^{2} \leq c$, we have by Jensen's inequality

$$
\left(\sum_{i=1}^{n-1}\left|\gamma_{i}\right| \psi_{i}(x)^{2}\right)^{p} \leq c \sum_{i=1}^{n-1}\left|\gamma_{i}\right|^{p} \psi_{i}(x)^{2} \leq c \sum_{i=1}^{n-1}\left|\gamma_{i}\right|^{p}
$$

and if $0<p<1$, then

$$
\left(\sum_{i=1}^{n-1}\left|\gamma_{i}\right| \psi_{i}(x)^{2}\right)^{p} \leq \sum_{i=1}^{n-1}\left|\gamma_{i}\right|^{p} \psi_{i}(x)^{2 p} \leq c \sum_{i=1}^{n-1}\left|\gamma_{i}\right|^{p}
$$

Therefore, using (2.7) we have

$$
\begin{aligned}
\left\|S_{n}-P_{n}\right\|_{p, w_{n}}^{p} & \leq c \int_{-1}^{1} \sum_{i=1}^{n-1}\left\|p_{i}-p_{i+1}\right\|_{\mathbb{L}_{p}\left(I_{i}\right)}^{p}\left|I_{i}\right|^{-1} w_{n}(x) \psi_{i}(x)^{(\mu-r-1) p} d x \\
& \leq c \int_{-1}^{1} \sum_{i=1}^{n-1}\left\|p_{i}-p_{i+1}\right\|_{\mathbb{L}_{p}\left(I_{i}\right)}^{p}\left|I_{i}\right|^{-1} w_{n}\left(x_{i}\right) \psi_{i}(x)^{(\mu-r-1) p-s} d x \\
& \leq c \sum_{i=1}^{n-1} \omega_{r}\left(f,\left|I_{i}\right|, J_{i} \cup J_{i+1}\right)_{p}^{p}\left|I_{i}\right|^{-1} w_{n}\left(x_{i}\right) \int_{-1}^{1} \psi_{i}(x)^{(\mu-r-1) p-s} d x .
\end{aligned}
$$

Now, if $\alpha \geq 2$, then $\int_{-1}^{1} \psi_{i}(x)^{\alpha} d x \leq c\left|I_{i}\right|$, and so

$$
\left\|S_{n}-P_{n}\right\|_{p, w_{n}}^{p} \leq c \sum_{i=1}^{n-1} w_{n}\left(x_{i}\right) \omega_{r}\left(f,\left|I_{i}\right|, J_{i} \cup J_{i+1}\right)_{p}^{p} \leq c \tilde{\omega}_{\varphi}^{r}(f, \theta / n)_{p, w_{n}}^{p} .
$$

provided $(\mu-r-1) p-s \geq 2$.

Now, note that

$$
P_{n}^{(\nu)}(x)=p_{n}^{(\nu)}(x)+\sum_{i=1}^{n-1} \sum_{l=0}^{\nu}\left(\begin{array}{l}
\nu \\
l
\end{array}\right)\left[p_{i}^{(l)}(x)-p_{i+1}^{(l)}(x)\right] T_{i}^{(\nu-l)}(x),
$$

and so, for $r \leq \nu \leq \nu_{0}$ (which guarantees that $p_{n}^{(\nu)} \equiv 0$ ), we have using Lemma 3.1 and 


$$
\begin{aligned}
\left\|\rho_{n}^{\nu} P_{n}^{(\nu)}\right\|_{p, w_{n}}^{p} & \leq \int_{-1}^{1} w_{n}(x) \rho_{n}(x)^{\nu p}\left[\sum_{i=1}^{n-1} \sum_{l=0}^{\nu}\left(\begin{array}{c}
\nu \\
l
\end{array}\right)\left|p_{i}^{(l)}(x)-p_{i+1}^{(l)}(x)\right| \cdot\left|T_{i}^{(\nu-l)}(x)\right|\right]^{p} d x \\
& \leq c \int_{-1}^{1} w_{n}(x) \rho_{n}(x)^{\nu p}\left[\sum_{i=1}^{n-1} \sum_{l=0}^{\nu}\left\|p_{i}^{(l)}-p_{i+1}^{(l)}\right\|_{\infty}\left|I_{i}\right|^{-\nu+l} \psi_{i}(x)^{\mu}\right]^{p} d x \\
& \leq c \int_{-1}^{1} w_{n}(x) \rho_{n}(x)^{\nu p}\left[\sum_{i=1}^{n-1} \sum_{l=0}^{\nu}\left\|p_{i}-p_{i+1}\right\|_{\mathbb{L}_{p}\left(I_{i}\right)}\left|I_{i}\right|^{-\nu-1 / p} \psi_{i}(x)^{\mu-r+l+1}\right]^{p} d x \\
& \leq c \int_{-1}^{1} w_{n}(x) \rho_{n}(x)^{\nu p}\left[\sum_{i=1}^{n-1}\left\|p_{i}-p_{i+1}\right\|_{\mathbb{L}_{p}\left(I_{i}\right)}\left|I_{i}\right|^{-\nu-1 / p} \psi_{i}(x)^{\mu-r+1}\right]^{p} d x \\
& \leq c \int_{-1}^{1} w_{n}(x) \rho_{n}(x)^{\nu p} \sum_{i=1}^{n-1}\left\|p_{i}-p_{i+1}\right\|_{\mathbb{L}_{p}\left(I_{i}\right)}^{p}\left|I_{i}\right|^{-\nu p-1} \psi_{i}(x)^{(\mu-r-1) p} d x \\
& \leq c \int_{-1}^{1} \rho_{n}(x)^{\nu p} \sum_{i=1}^{n-1}\left\|p_{i}-p_{i+1}\right\|_{\mathbb{L}_{p}\left(I_{i}\right)}^{p}\left|I_{i}\right|^{-\nu p-1} w_{n}\left(x_{i}\right) \psi_{i}(x)^{(\mu-r-1) p-s} d x .
\end{aligned}
$$

Now, since $\rho_{n}(x)^{2} \leq 4 \rho_{n}\left(x_{i}\right)\left(\left|x-x_{i}\right|+\rho_{n}\left(x_{i}\right)\right)$ and $\left|I_{i}\right| \sim \rho_{n}\left(x_{i}\right)$,

$$
\begin{aligned}
\left\|\rho_{n}^{\nu} P_{n}^{(\nu)}\right\|_{p, w_{n}}^{p} \leq & c \int_{-1}^{1} \sum_{i=1}^{n-1}\left\|p_{i}-p_{i+1}\right\|_{\mathbb{L}_{p}\left(I_{i}\right)}^{p}\left[\rho_{n}\left(x_{i}\right)\left(\left|x-x_{i}\right|+\rho_{n}\left(x_{i}\right)\right)\right]^{\nu p / 2} \\
& \times\left|I_{i}\right|^{-\nu p-1} w_{n}\left(x_{i}\right) \psi_{i}(x)^{(\mu-r-1) p-s} d x \\
\leq & c \int_{-1}^{1} \sum_{i=1}^{n-1}\left\|p_{i}-p_{i+1}\right\|_{\mathbb{L}_{p}\left(I_{i}\right)}^{p}\left|I_{i}\right|^{-1} w_{n}\left(x_{i}\right) \psi_{i}(x)^{(\mu-r-1-\nu / 2) p-s} d x,
\end{aligned}
$$

and exactly the same sequence of inequalities as above yields

$$
\left\|\rho_{n}^{\nu} P_{n}^{(\nu)}\right\|_{p, w_{n}}^{p} \leq c \tilde{\omega}_{\varphi}^{r}(f, \theta / n)_{p, w_{n}}^{p}
$$

provided $\left(\mu-r-1-\nu_{0} / 2\right) p-s \geq 2$. Thus, if we pick $\mu=\mu\left(r, \nu_{0}, p, s\right)$ so that this (the most restrictive in this proof) inequality as well as the restrictions on $\mu$ from Lemma 3.1 are satisfied then, for each $n \in \mathbb{N}$, we have constructed a polynomial $\tilde{P}_{n}$ of degree $<c_{*} n$ with some $c_{*} \in \mathbb{N}$ depending only on $r, \nu_{0}, p$ and $s$, such that

$$
\left\|f-\tilde{P}_{n}\right\|_{p, w_{n}} \leq c \tilde{\omega}_{\varphi}^{r}(f, \theta / n)_{p, w_{n}}^{p}
$$

and

$$
\left\|\rho_{n}^{\nu} \tilde{P}_{n}^{(\nu)}\right\|_{p, w_{n}} \leq c \tilde{\omega}_{\varphi}^{r}(f, \theta / n)_{p, w_{n}}, \quad r \leq \nu \leq \nu_{0}
$$

We now pick $\theta:=\vartheta /\left(2 c_{*}\right)$, and conclude that this completes the proof for $n \geq c_{*}$. Indeed, suppose that $n \geq c_{*}$. Then there exists $m \in \mathbb{N}$ such that $m c_{*} \leq n<(m+1) c_{*}$. Then, for polynomials $\tilde{\widetilde{P}}_{m}$ of degree $<c_{*} m$ (which implies that $\tilde{P}_{m} \in \Pi_{n}$ ), (5.5) and (5.6) hold, and

$$
\tilde{\omega}_{\varphi}^{r}(f, \theta / m)_{p, w_{m}}=\tilde{\omega}_{\varphi}^{r}\left(f, \vartheta /\left(2 c_{*} m\right)\right)_{p, w_{m}} \leq c \tilde{\omega}_{\varphi}^{r}(f, \vartheta / n)_{p, w_{m}} \leq c \tilde{\omega}_{\varphi}^{r}(f, \vartheta / n)_{p, w_{n}}
$$


since $w_{n}(x) \sim w_{m}(x)$ if $n \sim m$. Also, $\rho_{n}(x) \sim \rho_{m}(x)$ if $n \sim m$, and the proof is complete for $n \geq c_{*}$. Finally, for $r \leq n \leq c_{*}$, the statement of the theorem follows from the case $n=r$, Whitney's inequality (5.1) and the observation that $w_{r}(0) \omega_{r}(f, 2)_{p}^{p} \leq$ $c \tilde{\omega}_{\varphi}^{r}(f, \vartheta / r)_{p, w_{r}}^{p}$ (see Lemma 5.1). This completes the proof in the case $0<p<\infty$.

If $p=\infty$, the proof is analogous and, in fact, simpler. For completeness, we sketch it below.

The estimate $\left\|f-S_{n}\right\|_{\infty, w_{n}} \leq c \omega_{\varphi}^{r}(f, \theta / n)_{\infty, w_{n}}$ immediately follows from Lemma 5.2, and for each $x \in[-1,1]$, we have

$$
\begin{aligned}
w_{n}(x)\left|S_{n}(x)-P_{n}(x)\right| & \leq w_{n}(x) \sum_{i=1}^{n-1}\left|p_{i}(x)-p_{i+1}(x)\right| \cdot\left|\chi_{i}(x)-T_{i}(x)\right| \\
& \leq c \sum_{i=1}^{n-1}\left\|p_{i}-p_{i+1}\right\|_{\mathbb{C}\left(I_{i}\right)} w_{n}\left(x_{i}\right) \psi_{i}(x)^{\mu-r-s+1} \\
& \leq c \sum_{i=1}^{n-1} \omega_{r}\left(f,\left|I_{i}\right|, J_{i} \cup J_{i+1}\right)_{\infty} w_{n}\left(x_{i}\right) \psi_{i}(x)^{\mu-r-s+1} \\
& \leq c \omega_{\varphi}^{r}(f, \theta / n)_{\infty, w_{n}} \sum_{i=1}^{n-1} \psi_{i}(x)^{\mu-r-s+1} \\
& \leq c \omega_{\varphi}^{r}(f, \theta / n)_{\infty, w_{n}},
\end{aligned}
$$

provided $\mu-r-s+1 \geq 2$.

Similarly, for $r \leq \nu \leq \nu_{0}$, as in the case $p<\infty$, we have

$$
\begin{aligned}
\rho_{n}(x)^{\nu} w_{n}(x)\left|P_{n}^{(\nu)}(x)\right| & \leq c w_{n}(x) \rho_{n}(x)^{\nu} \sum_{i=1}^{n-1} \sum_{l=0}^{\nu}\left|p_{i}^{(l)}(x)-p_{i+1}^{(l)}(x)\right| \cdot\left|T_{i}^{(\nu-l)}(x)\right| \\
& \leq c w_{n}(x) \rho_{n}(x)^{\nu} \sum_{i=1}^{n-1}\left\|p_{i}-p_{i+1}\right\|_{\mathbb{C}\left(I_{i}\right)}\left|I_{i}\right|^{-\nu} \psi_{i}(x)^{\mu-r+1} \\
& \leq c \sum_{i=1}^{n-1}\left\|p_{i}-p_{i+1}\right\|_{\mathbb{C}\left(I_{i}\right)} w_{n}\left(x_{i}\right) \psi_{i}(x)^{\mu-r-s+1-\nu / 2} \\
& \leq c \omega_{\varphi}^{r}(f, \theta / n)_{\infty, w_{n}},
\end{aligned}
$$

if $\mu-r-s+1-\nu / 2 \geq 2$. This completes the proof for $n \geq c_{*} n$, and the rest of the proof is the same as in the case $p<\infty$ taking into account that $w_{r}(0) \omega_{r}(f, 2)_{\infty} \leq$ $c \omega_{\varphi}^{r}(f, \vartheta / r)_{\infty, w_{r}}$.

\section{Markov-Bernstein type theorems}

Lemma 6.1. Let $w$ be a doubling weight, $r \in \mathbb{N}$ and $0<p \leq \infty$. Then, for all $n \in \mathbb{N}$ and $P_{n} \in \Pi_{n}$,

$$
\left\|\rho_{n}^{r} P_{n}^{(r)}\right\|_{p, w_{n}} \leq c\left\|P_{n}\right\|_{p, w_{n}} .
$$

where the constant $c$ depends only on $r, p$ and the doubling constant of $w$. 
Lemma 6.1 will be used to prove an inverse theorem in the case $1 \leq p \leq \infty$. However, in the case $0<p<1$, it will not be sufficient and will have to be much improved since we will need to know the dependence of the constant $c$ in (6.1) on $r$ making sure that it does not grow too fast with $r$. This will be done in Section 6.1.

Proof. First, we recall Markov-Bernstein's inequality (see e.g. [17], [7, (7.2.7)], [9], [22, Lemma 4], [6, Lemma 2.2], [1, (A.4.22)], [18] as well (3.3))

$$
\left\|\rho_{n}^{r} P_{n}^{(r)}\right\|_{p} \leq c\left\|P_{n}\right\|_{p}, \quad P_{n} \in \Pi_{n} \quad \text { and } \quad 0<p \leq \infty
$$

where $c$ depends only on $r$ and $p$.

Clearly, (6.1) is true if $r=0$. Now, using strong induction in $r$, we suppose that it is true for all $0 \leq l \leq r-1$. Using Theorem 4.1 with $\nu_{0}=r+1$, for example (and noting that, in the case $p=\infty$, we take $1 / p$ to be 1 in (4.1) and (4.2)), the Leibniz formula and (6.2) we have

$$
\begin{aligned}
\left\|\rho_{n}^{r} P_{n}^{(r)}\right\|_{p, w_{n}} & \leq c\left\|\rho_{n}^{r} P_{n}^{(r)} \mathcal{Q}_{n}\right\|_{p} \leq c\left\|\rho_{n}^{r}\left(P_{n} Q_{n}\right)^{(r)}\right\|_{p}+c \sum_{l=0}^{r-1}\left\|\rho_{n}^{r} P_{n}^{(l)} \mathcal{Q}_{n}^{(r-l)}\right\|_{p} \\
& \leq c\left\|\rho_{n}^{r}\left(P_{n} Q_{n}\right)^{(r)}\right\|_{p}+c \sum_{l=0}^{r-1}\left\|\rho_{n}^{l} P_{n}^{(l)}\right\|_{p, w_{n}} \\
& \leq c\left\|P_{n} Q_{n}\right\|_{p}+c\left\|P_{n}\right\|_{p, w_{n}} \leq c\left\|P_{n}\right\|_{p, w_{n}},
\end{aligned}
$$

and so (6.1) is proved.

\subsection{A refinement of Lemma 6.1 for $0<p<1$}

In the proof of the inverse theorem in the case $0<p<1$, we will need to know the dependence of $c$ in Lemma 6.1 on $r$ making sure that it does not grow too fast with $r$ (since this estimate will be used for all $0 \leq r \leq n-1$ ). Hence, we need to reprove Lemma 6.1 in the case $0<p<1$ paying particular attention to the constants in all estimates.

It is convenient to denote

$$
\delta_{k}(x):=\max \left\{\frac{\sqrt{1-x^{2}}}{k}, \frac{1}{k^{2}}\right\} .
$$

We start with the following lemma.

Lemma 6.2. Let $0<p<1$. Then, for every $n, k \in \mathbb{N}, 0 \leq \mu \leq n-1, k \geq n / 2$, and $P_{n} \in \Pi_{n}$,

$$
\left\|\delta_{k}^{\mu+1} P_{n}^{\prime}\right\|_{p} \leq c(\mu+1)\left\|\delta_{k}^{\mu} P_{n}\right\|_{p}
$$

where the constant $c$ depends only on $p$ and is independent of $\mu, n$ and $k$.

In one form or another, Lemma 6.2 is known. For example, it follows from [22] and [6] (see also [18]). However, since this result and, in particular, the exact dependence of the constant on $\mu$ is crucial in our proofs and since, as far as we know, Lemma 6.2 was not explicitly stated anywhere in the present form we sketch its short (and rather standard) proof. 
Proof. It is known (see e.g. [22, (2.11)] or [6, (2.3)]) that, for any $n \in \mathbb{N}, 0 \leq \mu \leq n-1$ and $P_{n} \in \Pi_{n}$,

$$
\left\|\varphi^{\mu+1} P_{n}^{\prime}\right\|_{p} \leq c_{1} n(\mu+1)\left\|\varphi^{\mu} P_{n}\right\|_{p}, \quad c_{1}=c_{1}(p) .
$$

It is also well known that

$$
\left\|P_{n}^{\prime}\right\|_{p} \leq c_{2} n^{2}\left\|P_{n}\right\|_{p}, \quad c_{2}=c_{2}(p) .
$$

Therefore, denoting $\mathcal{E}_{k}:=\left\{x \mid \sqrt{1-x^{2}} \leq 1 / k\right\}$ and noting that $\delta_{k}(x)=1 / k^{2}$ if $x \in \mathcal{E}_{k}$, and $\delta_{k}(x)=\varphi(x) / k$ if $x \in[-1,1] \backslash \mathcal{E}_{k}$, we have

$$
\begin{aligned}
2^{1-1 / p}\left\|\delta_{k}^{\mu+1} P_{n}^{\prime}\right\|_{p} & \leq\left\|\delta_{k}^{\mu+1} P_{n}^{\prime}\right\|_{\mathbb{L}_{p}\left(\varepsilon_{k}\right)}+\left\|\delta_{k}^{\mu+1} P_{n}^{\prime}\right\|_{\mathbb{L}_{p}\left([-1,1] \backslash \varepsilon_{k}\right)} \\
& =k^{-2 \mu-2}\left\|P_{n}^{\prime}\right\|_{\mathbb{L}_{p}\left(\varepsilon_{k}\right)}+k^{-\mu-1}\left\|\varphi^{\mu+1} P_{n}^{\prime}\right\|_{\mathbb{L}_{p}\left([-1,1] \backslash \varepsilon_{k}\right)} \\
& \leq k^{-2 \mu-2}\left\|P_{n}^{\prime}\right\|_{p}+k^{-\mu-1}\left\|\varphi^{\mu+1} P_{n}^{\prime}\right\|_{p} \\
& \leq c_{2} k^{-2 \mu-2} n^{2}\left\|P_{n}\right\|_{p}+c_{1} n(\mu+1) k^{-\mu-1}\left\|\varphi^{\mu} P_{n}\right\|_{p} \\
& =c_{2}(n / k)^{2}\left\|k^{-2 \mu} P_{n}\right\|_{p}+c_{1}(n / k)(\mu+1)\left\|[\varphi / k]^{\mu} P_{n}\right\|_{p} \\
& \leq\left[c_{2}(n / k)^{2}+c_{1}(n / k)(\mu+1)\right]\left\|\delta_{k}^{\mu} P_{n}\right\|_{p} \\
& \leq 4\left(c_{1}+c_{2}\right)(\mu+1)\left\|\delta_{k}^{\mu} P_{n}\right\|_{p} .
\end{aligned}
$$

Lemma 6.3. Let $w$ be a doubling weight and $0<p<1$. Then, for all $n, m, k \in \mathbb{N}$ and $\mu \in \mathbb{R}$ such that

$$
m \leq k, \quad n \leq k \quad \text { and } \quad 0 \leq \mu \leq n-1
$$

and $P_{n} \in \Pi_{n}$,

$$
\left\|\delta_{k}^{\mu+1} P_{n}^{\prime}\right\|_{p, w_{m}} \leq(\mu+1) c_{*}\left\|\delta_{k}^{\mu} P_{n}\right\|_{p, w_{m}},
$$

where the constant $c_{*}$ depends only on $p$ and the doubling constant of $w$.

Proof. First, using Theorem 4.1 (with $\nu_{0}=1$ ) we let $Q_{m} \in \Pi_{m}$ be such that

$$
c_{1} w_{m}(x)^{1 / p} \leq Q_{m}(x) \leq c_{2} w_{m}(x)^{1 / p}
$$

and

$$
\left|\rho_{m}(x) Q_{m}^{\prime}(x)\right| \leq c_{3} w_{m}(x)^{1 / p},
$$

where constants $c_{1}, c_{2}$ and $c_{3}$ depend only on $p$ and the doubling constant of $w$.

Note that $P_{n} Q_{m} \in \Pi_{n+m-1}$ and so taking into account that $\mu \leq n-1 \leq n+m-2$ and $k \geq(n+m-1) / 2$, by Lemma 6.2, we have

$$
\left\|\delta_{k}^{\mu+1}\left(P_{n} Q_{m}\right)^{\prime}\right\|_{p} \leq c_{4}(\mu+1)\left\|\delta_{k}^{\mu} P_{n} Q_{m}\right\|_{p},
$$

where $c_{4}$ depends only on $p$. Therefore,

$$
\begin{aligned}
\left\|\delta_{k}^{\mu+1} P_{n}^{\prime}\right\|_{p, w_{m}} & \leq c_{1}^{-1}\left\|\delta_{k}^{\mu+1} P_{n}^{\prime} Q_{m}\right\|_{p} \\
& \leq c_{1}^{-1} 2^{-1+1 / p}\left(\left\|\delta_{k}^{\mu+1}\left(P_{n} Q_{m}\right)^{\prime}\right\|_{p}+\left\|\delta_{k}^{\mu+1} P_{n} Q_{m}^{\prime}\right\|_{p}\right) \\
& \leq c_{1}^{-1} 2^{-1+1 / p}\left(c_{4}(\mu+1)\left\|\delta_{k}^{\mu}\left(P_{n} Q_{m}\right)\right\|_{p}+c_{3}\left\|\delta_{k}^{\mu+1} \rho_{m}^{-1} P_{n} w_{m}^{1 / p}\right\|_{p}\right) \\
& \leq c_{1}^{-1} 2^{-1+1 / p}\left(c_{2} c_{4}(\mu+1)\left\|\delta_{k}^{\mu} P_{n}\right\|_{p, w_{m}}+c_{3}\left\|\delta_{k}^{\mu} P_{n}\right\|_{p, w_{m}}\right) \\
& \leq c_{1}^{-1} 2^{-1+1 / p}\left(c_{2} c_{4}+c_{3}\right)(\mu+1)\left\|\delta_{k}^{\mu} P_{n}\right\|_{p, w_{m}} .
\end{aligned}
$$


Corollary 6.4. Let $w$ be a doubling weight and $0<p<1$. Then, for all $n, m, k, r \in \mathbb{N}$ and $l \in \mathbb{N}_{0}$ such that $m \leq k, n \leq k, l \leq r \leq n-1$, and $P_{n} \in \Pi_{n}$,

$$
\left\|\delta_{k}^{r} P_{n}^{(r)}\right\|_{p, w_{m}} \leq\left(c_{*}\right)^{r-l} \frac{r !}{l !}\left\|\delta_{k}^{l} P_{n}^{(l)}\right\|_{p, w_{m}},
$$

where the constant $c_{*}$ depends only on $p$ and the doubling constant of $w$.

Proof. Lemma 6.3 implies

$\left\|\delta_{k}^{j+1} P_{n}^{(j+1)}\right\|_{p, w_{m}}=\left\|\delta_{k}^{j+1}\left(P_{n}^{(j)}\right)^{\prime}\right\|_{p, w_{m}} \leq(j+1) c(p)\left\|\delta_{k}^{j} P_{n}^{(j)}\right\|_{p, w_{m}}, \quad$ for all $0 \leq j \leq r-1$,

which immediately implies the statement of the corollary.

Now, taking into account that $\delta_{n}(x) \leq \rho_{n}(x) \leq 2 \delta_{n}(x)$, we finally get the result that we need in order to prove the inverse type theorems for $0<p<1$.

Corollary 6.5. Let $w$ be a doubling weight, $0<p<1, n, r \in \mathbb{N}, l \in \mathbb{N}_{0}, 0 \leq l \leq r \leq$ $n-1$, and $P_{n} \in \Pi_{n}$. Then

$$
\left\|\rho_{n}^{r} P_{n}^{(r)}\right\|_{p, w_{n}} \leq 2^{l}\left(c_{*}\right)^{r-l} \frac{r !}{l !}\left\|\rho_{n}^{l} P_{n}^{(l)}\right\|_{p, w_{n}}
$$

where the constant $c_{*}$ depends only on $p$ and the doubling constant of $w$.

We note that exactly the same proof as above (and actually simpler since Lemma 6.2 is no longer needed and (6.4) can be used) yields the following result.

Corollary 6.6. Let $w$ be a doubling weight, $0<p<1, n, r \in \mathbb{N}, l \in \mathbb{N}_{0}, 0 \leq l \leq r \leq$ $n-1$, and $P_{n} \in \Pi_{n}$. Then

$$
\left\|\varphi^{r} P_{n}^{(r)}\right\|_{p, w_{n}} \leq\left(c_{*}\right)^{r-l} \frac{r !}{l !} n^{r-l}\left\|\varphi^{l} P_{n}^{(l)}\right\|_{p, w_{n}}
$$

where the constant $c_{*}$ depends only on $p$ and the doubling constant of $w$.

\subsection{Other Markov-Bernstein type estimates in the case $0<p<1$}

Lemma 6.7. Let $0<p<1$ and $n, m, r \in \mathbb{N}$ be such that $m \leq n$, and suppose that $w$ is a doubling weight from the class $\mathcal{W}_{\Lambda}^{\delta, \gamma}$ with $\gamma \leq r p$.

Then, for any $\kappa>0$, there exists a positive constant $\vartheta$ depending only on $\kappa, r, p, \Lambda$, and the doubling constant of $w$, such that, for any $P_{m} \in \Pi_{m}$ and $0<t \leq 1 / m$,

$$
\omega_{\varphi}^{r}\left(P_{m}, \vartheta t\right)_{p, w_{n}} \leq \kappa\left(\frac{n}{m}\right)^{\delta / p}(t m)^{r}\left\|\rho_{m}^{r} P_{m}^{(r)}\right\|_{p, w_{m}} .
$$

The following corollary is an immediate consequence of Lemma 6.7 and Corollary 6.5 with $l=0$ (by setting $\kappa:=\left[\left(c_{*}\right)^{r} r !\right]^{-1}$, where $c_{*}$ is the constant from Corollary [6.5).

Corollary 6.8. Let $0<p<1$ and $n, m, r \in \mathbb{N}$ be such that $m \leq n$, and suppose that $w$ is a doubling weight from the class $\mathcal{W}_{\Lambda}^{\delta, \gamma}$ with $\gamma \leq r p$.

Then, there exists a positive constant $\vartheta$ depending only on $r, p, \Lambda$, and the doubling constant of $w$, such that, for any $P_{m} \in \Pi_{m}$ and $0<t \leq 1 / m$,

$$
\omega_{\varphi}^{r}\left(P_{m}, \vartheta t\right)_{p, w_{n}} \leq\left(\frac{n}{m}\right)^{\delta / p}(t m)^{r}\left\|P_{m}\right\|_{p, w_{m}} .
$$


Proof of Lemma 6.7. The method of the proof is rather standard (see e.g. [2, 5, 22]). Suppose that $h \leq \vartheta t \leq \vartheta / m$, where $\vartheta$ is a positive constant that we will choose later. Using Taylor's expansion of $P_{m}$ we have

$$
\begin{aligned}
\Delta_{h \varphi(x)}^{r}\left(P_{m}, x\right) & =\sum_{i=0}^{r}\left(\begin{array}{c}
r \\
i
\end{array}\right)(-1)^{r-i} P_{m}(x+(i-r / 2) h \varphi(x)) \\
& =\sum_{i=0}^{r}\left(\begin{array}{c}
r \\
i
\end{array}\right)(-1)^{r-i} \sum_{j=0}^{m-1} \frac{(i-r / 2)^{j} h^{j}}{j !} \varphi(x)^{j} P_{m}^{(j)}(x) \\
& =\sum_{j=0}^{m-1} \varphi(x)^{j} P_{m}^{(j)}(x) \frac{h^{j}}{j !} \sum_{i=0}^{r}\left(\begin{array}{c}
r \\
i
\end{array}\right)(-1)^{r-i}(i-r / 2)^{j} \\
& =\sum_{j=0}^{m-1} \varphi(x)^{j} P_{m}^{(j)}(x) \frac{h^{j}}{j !} \Delta_{1}^{r}\left((\cdot)^{j}, 0\right) .
\end{aligned}
$$

Recall now that, if $g^{(r)}$ is continuous on $[x-r \mu / 2, x+r \mu / 2]$ then, for some $\xi \in(x-$ $r \mu / 2, x+r \mu / 2)$,

$$
\Delta_{\mu}^{r}(g, x)=\mu^{r} g^{(r)}(\xi) .
$$

This implies

$$
\left|\Delta_{1}^{r}\left((\cdot)^{j}, 0\right)\right| \leq \begin{cases}0, & \text { if } 0 \leq j \leq r-1, \\ \frac{j !}{(j-r) !}(r / 2)^{j-r}, & \text { if } j \geq r .\end{cases}
$$

Also, since $w \in \mathcal{W}_{\Lambda}^{\delta, \gamma}$,

$$
w_{n}(x) \varphi(x)^{\gamma} \leq \Lambda n^{\delta} m^{\gamma-\delta} \rho_{m}(x)^{\gamma} w_{m}(x),
$$

and taking into account that $j p \geq r p \geq \gamma, r \leq j \leq m-1$, and $\varphi(x) \leq m \rho_{m}(x)$, we have

$$
\begin{aligned}
\| & \Delta_{h \varphi}^{r}\left(P_{m}\right) \|_{p, w_{n}}^{p} \\
& =\int_{-1}^{1} w_{n}(x)\left|\Delta_{h \varphi(x)}^{r}\left(P_{m}, x\right)\right|^{p} d x \\
& \leq \sum_{j=r}^{m-1}\left(\frac{h^{j}}{(j-r) !}(r / 2)^{j-r}\right)^{p} \int_{-1}^{1} w_{n}(x)\left|\varphi(x)^{j} P_{m}^{(j)}(x)\right|^{p} d x \\
& \leq \Lambda \sum_{j=r}^{m-1}\left(\frac{h^{j}}{(j-r) !}(r / 2)^{j-r}\right)^{p} \int_{-1}^{1} n^{\delta} m^{\gamma-\delta} \rho_{m}(x)^{\gamma} \varphi(x)^{j p-\gamma} w_{m}(x)\left|P_{m}^{(j)}(x)\right|^{p} d x \\
& \leq \Lambda\left(\frac{n}{m}\right)^{\delta} \sum_{j=r}^{m-1}\left(\frac{(h m)^{j}}{(j-r) !}(r / 2)^{j-r}\right)^{p} \int_{-1}^{1} w_{m}(x)\left|\rho_{m}(x)^{j} P_{m}^{(j)}(x)\right|^{p} d x .
\end{aligned}
$$

It follows from Corollary 6.5 that, for some constant $c_{1}$ that depends only on $p$ and the doubling constant of $w$,

$$
\begin{aligned}
& \int_{-1}^{1} w_{m}(x)\left|\rho_{m}(x)^{j} P_{m}^{(j)}(x)\right|^{p} d x=\left\|\rho_{m}^{j} P_{m}^{(j)}\right\|_{p, w_{m}}^{p} \\
& \quad \leq\left(2^{r}\left(c_{1}\right)^{j-r} \frac{j !}{r !}\right)^{p}\left\|\rho_{m}^{r} P_{m}^{(r)}\right\|_{p, w_{m}}^{p}, \quad r \leq j \leq m-1 .
\end{aligned}
$$


Therefore, recalling that $h \leq \vartheta / m$, we have

$$
\begin{aligned}
\left\|\Delta_{h \varphi}^{r}\left(P_{m}\right)\right\|_{p, w_{n}}^{p} & \leq \Lambda\left(\frac{n}{m}\right)^{\delta} \sum_{j=r}^{m-1}\left(\frac{(h m)^{j} j !}{(j-r) ! r !}(r / 2)^{j-r} 2^{r}\left(c_{1}\right)^{j-r}\right)^{p}\left\|\rho_{m}^{r} P_{m}^{(r)}\right\|_{p, w_{m}}^{p} \\
& \leq \Lambda 2^{r p}\left(\frac{n}{m}\right)^{\delta}\left\|\rho_{m}^{r} P_{m}^{(r)}\right\|_{p, w_{m}}^{p}(h m)^{r p} \sum_{j=r}^{m-1}\left(\left(\begin{array}{l}
j \\
r
\end{array}\right)\left(\vartheta r c_{1} / 2\right)^{j-r}\right)^{p} .
\end{aligned}
$$

Now, if $\vartheta \leq 1 /\left(r c_{1}\right)$, noting that $\sum_{j=r}^{\infty}(1 / 2)^{(j-r) p}\left[\left(\begin{array}{l}j \\ r\end{array}\right)\right]^{p}=c_{2}$, where $c_{2}$ depends only on $r$ and $p$, we conclude that

$$
\omega_{\varphi}^{r}\left(P_{m}, \vartheta t\right)_{p, w_{n}} \leq 2^{r}\left(\Lambda c_{2}\right)^{1 / p} \vartheta^{r}\left(\frac{n}{m}\right)^{\delta / p}(t m)^{r}\left\|\rho_{m}^{r} P_{m}^{(r)}\right\|_{p, w_{m}} .
$$

Hence, if we guarantee that $\vartheta$ is such that $2^{r}\left(\Lambda c_{2}\right)^{1 / p} \vartheta^{r} \leq \kappa$, then

$$
\omega_{\varphi}^{r}\left(P_{m}, \vartheta t\right)_{p, w_{n}} \leq \kappa\left(\frac{n}{m}\right)^{\delta / p}(t m)^{r}\left\|\rho_{m}^{r} P_{m}^{(r)}\right\|_{p, w_{m}}
$$

and the proof is complete if we pick $\vartheta:=\min \left\{1 /\left(r c_{1}\right), \kappa^{1 / r}\left(\Lambda c_{2}\right)^{-1 /(r p)} / 2\right\}$.

Note now that if the same weight $w_{n}$ is used on both sides of (6.5) (i.e., $m=n$ ), then there is no need to use (6.9) in the proof of Lemma 6.7. Also, one can keep using $\varphi^{j p}$ and not replace it by $\left(n \rho_{n}\right)^{j p}$, and use Corollary 6.6 instead of Corollary 6.5 in order to estimate $\left\|\varphi^{j} P_{n}^{(j)}\right\|_{p, w_{n}}$. The following result that is proved using an idea from [5] will be used in the last section to show the equivalence of the moduli and certain realization functionals. Even though the proof is very similar to that of Lemma 6.7 we sketch it below for completeness.

Lemma 6.9. Let $w$ be a doubling weight, $0<p<1$ and $n, r \in \mathbb{N}$. Then, there exists a positive constant $\vartheta$ depending only on $r, p$ and the doubling constant of $w$, such that, for any $P_{n} \in \Pi_{n}$ and $0<h \leq t \leq \vartheta / n$,

$$
(1 / 2)^{1 / p} h^{r}\left\|\varphi^{r} P_{n}^{(r)}\right\|_{p, w_{n}} \leq\left\|\Delta_{h \varphi}^{r}\left(P_{n}\right)\right\|_{p, w_{n}} \leq(3 / 2)^{1 / p} h^{r}\left\|\varphi^{r} P_{n}^{(r)}\right\|_{p, w_{n}},
$$

and so

$$
(1 / 2)^{1 / p} t^{r}\left\|\varphi^{r} P_{n}^{(r)}\right\|_{p, w_{n}} \leq \omega_{\varphi}^{r}\left(P_{n}, t\right)_{p, w_{n}} \leq(3 / 2)^{1 / p} t^{r}\left\|\varphi^{r} P_{n}^{(r)}\right\|_{p, w_{n}} .
$$

Proof. The beginning of the proof is similar to that of Lemma 6.7. We suppose that $h \leq t \leq \vartheta / n$, where $\vartheta$ is a positive constant that we will choose later. Then using (6.7) and (6.8), and taking into account that $\Delta_{1}^{r}\left((\cdot)^{r}, 0\right)=r$ !, we have

$$
\left\|\Delta_{h \varphi}^{r}\left(P_{n}\right)-h^{r} \varphi^{r} P_{n}^{(r)}\right\|_{p, w_{n}}^{p} \leq \sum_{j=r+1}^{n-1}\left(\frac{h^{j}}{(j-r) !}(r / 2)^{j-r}\right)^{p} \int_{-1}^{1} w_{n}(x)\left|\varphi(x)^{j} P_{n}^{(j)}(x)\right|^{p} d x .
$$

Using Corollary 6.6 we conclude that, for some constant $c_{1}$ that depends only on $p$ and the doubling constant of $w$,

$$
\begin{aligned}
& \int_{-1}^{1} w_{n}(x)\left|\varphi(x)^{j} P_{n}^{(j)}(x)\right|^{p} d x=\left\|\varphi^{j} P_{n}^{(j)}\right\|_{p, w_{n}}^{p} \\
& \quad \leq\left(\left(c_{1}\right)^{j-r} \frac{j !}{r !}\right)^{p} n^{(j-r) p}\left\|\varphi^{r} P_{n}^{(r)}\right\|_{p, w_{n}}^{p}, \quad r+1 \leq j \leq n-1 .
\end{aligned}
$$


Therefore, recalling that $h \leq \vartheta / n$, we have

$$
\begin{aligned}
\left\|\Delta_{h \varphi}^{r}\left(P_{n}\right)-h^{r} \varphi^{r} P_{n}^{(r)}\right\|_{p, w_{n}}^{p} & \leq \sum_{j=r+1}^{n-1}\left(\frac{h^{j}}{(j-r) !}(r / 2)^{j-r}\left(c_{1}\right)^{j-r} \frac{j !}{r !}\right)^{p} n^{(j-r) p}\left\|\varphi^{r} P_{n}^{(r)}\right\|_{p, w_{n}}^{p} \\
& \leq h^{r p}\left\|\varphi^{r} P_{n}^{(r)}\right\|_{p, w_{n}}^{p} \sum_{j=r+1}^{n-1}\left(\left(\vartheta r c_{1} / 2\right)^{j-r}\left(\begin{array}{l}
j \\
r
\end{array}\right)\right)^{p} \\
& \leq h^{r p}\left\|\varphi^{r} P_{n}^{(r)}\right\|_{p, w_{n}}^{p}\left(\vartheta r c_{1} / 2\right)^{p} \sum_{j=r+1}^{n-1}\left(\left(\vartheta r c_{1} / 2\right)^{j-r-1}\left(\begin{array}{l}
j \\
r
\end{array}\right)\right)^{p} .
\end{aligned}
$$

Now, if $\vartheta \leq 1 /\left(r c_{1}\right)$, then $\sum_{j=r+1}^{\infty}(1 / 2)^{(j-r-1) p}\left[\left(\begin{array}{l}j \\ r\end{array}\right)\right]^{p}=c_{2}$, where $c_{2}$ depends only on $r$ and $p$, and if $\vartheta \leq 2\left(2 c_{2}\right)^{-1 / p}\left(r c_{1}\right)^{-1}$, then we get

$$
\left\|\Delta_{h \varphi}^{r}\left(P_{n}\right)-h^{r} \varphi^{r} P_{n}^{(r)}\right\|_{p, w_{n}}^{p} \leq \frac{1}{2} h^{r p}\left\|\varphi^{r} P_{n}^{(r)}\right\|_{p, w_{n}}^{p}
$$

Therefore, if we set $\vartheta:=\min \left\{1 /\left(r c_{1}\right), 2\left(2 c_{2}\right)^{-1 / p}\left(r c_{1}\right)^{-1}\right\}$, then we get

$$
\frac{1}{2} h^{r p}\left\|\varphi^{r} P_{n}^{(r)}\right\|_{p, w_{n}}^{p} \leq\left\|\Delta_{h \varphi}^{r}\left(P_{n}\right)\right\|_{p, w_{n}}^{p} \leq \frac{3}{2} h^{r p}\left\|\varphi^{r} P_{n}^{(r)}\right\|_{p, w_{n}}^{p} .
$$

\section{Weighted polynomial approximation: inverse the- orems}

\subsection{Auxiliary results}

Lemma 7.1. If $w$ is a doubling weight, $0<p \leq \infty, f \in \mathbb{L}_{p}[-1,1], n, r \in \mathbb{N}, c_{*}>0$, and $t \leq c_{*} / n$, then

$$
\omega_{\varphi}^{r}(f, t)_{p, w_{n}} \leq c\|f\|_{p, w_{n}}
$$

where $c$ depends only on $r, c_{*}, p$, and the doubling constant of $w$.

Proof. First, we recall that $\Delta_{h \varphi(x)}^{r}(f, x)=0$ if $x \notin \mathfrak{D}_{r h / 2}$, where

$$
\mathfrak{D}_{\lambda}:=\{x \mid x \neq \pm 1 \quad \text { and } \quad x \pm \lambda \varphi(x) \in[-1,1]\}=\left\{x|| x \mid \leq \frac{1-\lambda^{2}}{1+\lambda^{2}}\right\}
$$

and hence, for $0<p<\infty$,

$$
\begin{aligned}
\omega_{\varphi}^{r}(f, t)_{p, w_{n}}^{p} & \leq c \sup _{0<h \leq t} \int_{\mathfrak{D}_{r h / 2}} w_{n}(x)\left(\sum_{i=0}^{r}\left(\begin{array}{l}
r \\
i
\end{array}\right)|f(x+(i-r / 2) h \varphi(x))|\right)^{p} d x \\
& \leq c \sup _{0<h \leq t} \sum_{i=0}^{r} \int_{\mathfrak{D}_{r h / 2}} w_{n}(x)|f(x+(i-r / 2) h \varphi(x))|^{p} d x .
\end{aligned}
$$


It is clear that, if $h \leq t \leq c / n$, then $h \varphi(x) \leq c \rho_{n}(x)$. Therefore, if $y_{i}(x):=x+(i-$ $r / 2) h \varphi(x), 0 \leq i \leq r$, then $\left|x-y_{i}(x)\right| \leq r h \varphi(x) / 2 \leq c \rho_{n}(x)$ and (2.2) implies that $w_{n}(x) \sim w_{n}\left(y_{i}(x)\right)$. Hence,

$$
\begin{aligned}
\omega_{\varphi}^{r}(f, t)_{p, w_{n}}^{p} & \leq c \sup _{0<h \leq t} \sum_{i=0}^{r} \int_{\mathfrak{D}_{r h / 2}} w_{n}\left(y_{i}(x)\right)\left|f\left(y_{i}(x)\right)\right|^{p} d x \\
& \leq c \int_{-1}^{1} w_{n}(y)|f(y)|^{p} d y \leq c\|f\|_{p, w_{n}}^{p} .
\end{aligned}
$$

In the case $p=\infty$, the needed modifications in the proof are obvious.

Lemma 7.2. Let $w$ be a doubling weight, $n, r \in \mathbb{N}, c_{*}>0, t \leq c_{*} / n, 1 \leq p \leq \infty$. If $f$ has the $(r-1)$ st locally absolutely continuous derivative on $(-1,1)$ and $\left\|\varphi^{r} f^{(r)}\right\|_{p}<\infty$, then

$$
\omega_{\varphi}^{r}(f, t)_{p, w_{n}} \leq c t^{r}\left\|\varphi^{r} f^{(r)}\right\|_{p, w_{n}},
$$

where $c$ depends only on $r, c_{*}, p$, and the doubling constant of $w$.

We remark that it is well known that, in general, Lemma 7.2 is not true for $0<p<1$ and, in fact, one can show that, for every $M \in \mathbb{R}$ and $n \in \mathbb{N}$, there exists an absolutely continuous function $f$ on $[-1,1]$ such that $E_{n}(f,[-1,1])_{p}>M\left\|f^{\prime}\right\|_{p}$.

Proof. If $f$ has the $(r-1)$ st absolutely continuous derivative, then

$$
\Delta_{h}^{r}(f, x)=\int_{-h / 2}^{h / 2} \ldots \int_{-h / 2}^{h / 2} f^{(r)}\left(x+t_{1}+\cdots+t_{r}\right) d t_{r} \ldots d t_{1} .
$$

In the case $1 \leq p<\infty$, if $h \leq c / n$, we have

$$
\begin{aligned}
& \left(\int_{-1}^{1} w_{n}(x)\left|\Delta_{h \varphi(x)}^{r}(f, x)\right|^{p} d x\right)^{1 / p} \\
& \leq\left(\int_{\mathfrak{D}_{r h / 2}}\left[\int_{-h \varphi(x) / 2}^{h \varphi(x) / 2} \cdots \int_{-h \varphi(x) / 2}^{h \varphi(x) / 2} w_{n}^{1 / p}(x)\left|f^{(r)}\left(x+t_{1}+\cdots+t_{r}\right)\right| d t_{r} \ldots d t_{1}\right]^{p} d x\right)^{1 / p} \\
& \leq c\left(\int _ { \mathfrak { D } _ { r h / 2 } } \left[\int_{-h \varphi(x) / 2}^{h \varphi(x) / 2} \cdots \int_{-h \varphi(x) / 2}^{h \varphi(x) / 2} w_{n}^{1 / p}\left(x+t_{1}+\cdots+t_{r}\right)\right.\right. \\
& \left.\left.\quad \times\left|f^{(r)}\left(x+t_{1}+\cdots+t_{r}\right)\right| d t_{r} \ldots d t_{1}\right]^{p} d x\right)^{1 / p} .
\end{aligned}
$$

By Hölder's inequality, for each $u$ satisfying $-1<x+u-h \varphi(x) / 2<x+u+h \varphi(x) / 2<1$, we have

$$
\begin{aligned}
\int_{-h \varphi(x) / 2}^{h \varphi(x) / 2} w_{n}^{1 / p}\left(x+u+t_{r}\right)\left|f^{(r)}\left(x+u+t_{r}\right)\right| d t_{r} & =\int_{x+u-h \varphi(x) / 2}^{x+u+h \varphi(x) / 2} w_{n}^{1 / p}(v)\left|f^{(r)}(v)\right| d v \\
& \leq\left\|w_{n}^{1 / p} \varphi^{r} f^{(r)}\right\|_{\mathbb{L}_{p}(\mathcal{A}(x, u))}\left\|\varphi^{-r}\right\|_{\mathbb{L}_{p^{\prime}}(\mathcal{A}(x, u))},
\end{aligned}
$$

where $1 / p+1 / p^{\prime}=1$ and

$$
\mathcal{A}(x, u):=[x+u-h \varphi(x) / 2, x+u+h \varphi(x) / 2] .
$$


The needed estimate now follows from

$$
\begin{aligned}
\int_{\mathfrak{D}_{r h / 2}} & {\left[\int_{-h \varphi(x) / 2}^{h \varphi(x) / 2} \cdots \int_{-h \varphi(x) / 2}^{h \varphi(x) / 2}\left\|\varphi^{-r}\right\|_{\mathbb{L}_{p^{\prime}}\left(\mathcal{A}\left(x, t_{1}+\cdots+t_{r-1}\right)\right)}\right.} \\
\times & \left.\left\|w_{n}^{1 / p} \varphi^{r} f^{(r)}\right\|_{\mathbb{L}_{p}\left(\mathcal{A}\left(x, t_{1}+\cdots+t_{r-1}\right)\right)} d t_{r-1} \ldots d t_{1}\right]^{p} d x \leq c h^{r p}\left\|w_{n}^{1 / p} \varphi^{r} f^{(r)}\right\|_{p}^{p},
\end{aligned}
$$

where $1 \leq p<\infty$. In the case $p=\infty$, an analogous sequence of estimates yields

$$
\begin{aligned}
& \sup _{x \in \mathfrak{D}_{r h / 2}} \int_{-h \varphi(x) / 2}^{h \varphi(x) / 2} \cdots \int_{-h \varphi(x) / 2}^{h \varphi(x) / 2}\left\|\varphi^{-r}\right\|_{\mathbb{L}_{1}\left(\mathcal{A}\left(x, t_{1}+\cdots+t_{r-1}\right)\right)} \\
& \quad \times\left\|w_{n} \varphi^{r} f^{(r)}\right\|_{\mathbb{L}_{\infty}\left(\mathcal{A}\left(x, t_{1}+\cdots+t_{r-1}\right)\right)} d t_{r-1} \ldots d t_{1} \leq c h^{r}\left\|w_{n} \varphi^{r} f^{(r)}\right\|_{\infty} .
\end{aligned}
$$

Note that, in the case $r=1$, estimates (7.1) and (7.2) are understood, respectively, as

$$
\int_{\mathfrak{D}_{h / 2}}\left\|\varphi^{-1}\right\|_{\mathbb{L}_{p^{\prime}}(\mathcal{A}(x, 0))}^{p}\left\|w_{n}^{1 / p} \varphi f^{\prime}\right\|_{\mathbb{L}_{p}(\mathcal{A}(x, 0))}^{p} d x \leq c h^{p}\left\|w_{n}^{1 / p} \varphi f^{\prime}\right\|_{p}^{p}, \quad 1 \leq p<\infty,
$$

and

$$
\sup _{x \in \mathfrak{D}_{h / 2}}\left\|\varphi^{-1}\right\|_{\mathbb{L}_{1}(\mathcal{A}(x, 0))}\left\|w_{n} \varphi f^{\prime}\right\|_{\mathbb{L}_{\infty}(\mathcal{A}(x, 0))} \leq \operatorname{ch}\left\|w_{n} \varphi f^{\prime}\right\|_{\infty}, \quad p=\infty .
$$

Estimates (7.1)-(7.4) were proved in [12] (see (4.2)-(4.4) there with $r=0$, variable " $k$ " replaced by " $r$ ", $g^{(r)}$ replaced by $w_{n}^{1 / p} f^{(r)}$ with $1 / \infty:=1$, and noting that $\mathfrak{D}_{\lambda}$ in [12] is actually $\mathfrak{D}_{\lambda / 2}$ in the current paper).

\subsection{Inverse theorem: the case $1 \leq p \leq \infty$}

Recall the following notation that was used in the introduction

$$
\lambda_{p}:= \begin{cases}p, & \text { if } p<\infty \\ 1, & \text { if } p=\infty\end{cases}
$$

Theorem 7.3. Let $r \in \mathbb{N}, 1 \leq p \leq \infty$, and $f \in \mathbb{L}_{p}[-1,1]$. Suppose that $w$ is a doubling weight from the class $\mathcal{W}_{\Lambda}^{\delta, \gamma}$ with $\gamma \leq r \lambda_{p}$. Then

$$
\omega_{\varphi}^{r}\left(f, n^{-1}\right)_{p, w_{n}} \leq \frac{c}{n^{r-\delta / \lambda_{p}}} \sum_{k=1}^{n} k^{r-1-\delta / \lambda_{p}} E_{k}(f)_{p, w_{k}},
$$

where the constant $c$ depends only on $r, p, \delta, \gamma, \Lambda$, and the doubling constant of the weight $w$.

Taking into account that any doubling weight belongs to the class $\mathcal{W}_{1}^{1,1}$ (see Remark 2.3) and that $\gamma=1 \leq r \lambda_{p}$, for all $r \in \mathbb{N}$ and $1 \leq p \leq \infty$, we immediately get the following corollary of Theorem 7.3 ,

Corollary 7.4. Let $w$ be a doubling weight, $r \in \mathbb{N}, 1 \leq p \leq \infty$, and $f \in \mathbb{L}_{p}[-1,1]$. Then

$$
\omega_{\varphi}^{r}\left(f, n^{-1}\right)_{p, w_{n}} \leq \frac{c}{n^{r-1 / \lambda_{p}}} \sum_{k=1}^{n} k^{r-1-1 / \lambda_{p}} E_{k}(f)_{p, w_{k}},
$$

the constant $c$ depends only on $r, p$ and the doubling constant of $w$. 
Remark 7.5. Since any weight that satisfies the $A^{*}$ property is in $\mathcal{W}^{0,0}$ (see Remark 2.4), it immediately follows from Theorem 7.3 that, for $A^{*}$ weights $w$, we have

$$
\omega_{\varphi}^{r}\left(f, n^{-1}\right)_{p, w_{n}} \leq \frac{c}{n^{r}} \sum_{k=1}^{n} k^{r-1} E_{k}(f)_{p, w_{k}}, \quad 1 \leq p \leq \infty .
$$

In the case $p=\infty$, this is the "inverse" part of [13, Theorem 1.3].

Proof of Theorem 7.3. Let $P_{n}^{*} \in \Pi_{n}$ denote a polynomial of (near) best approximation to $f$ with weight $w_{n}$, i.e.,

$$
c\left\|f-P_{n}^{*}\right\|_{p, w_{n}} \leq \inf _{P_{n} \in \Pi_{n}}\left\|f-P_{n}\right\|_{p, w_{n}}=E_{n}(f)_{p, w_{n}} .
$$

We let $N \in \mathbb{N}$ be such that $2^{N} \leq n<2^{N+1}$ and denote $m_{j}:=2^{j}$. Then recalling that $w_{n}(x) \sim w_{m}(x)$ if $n \sim m$, and using Lemma 7.1 we have

$$
\begin{aligned}
\omega_{\varphi}^{r}\left(f, n^{-1}\right)_{p, w_{n}} & \leq \omega_{\varphi}^{r}\left(f, 2^{-N}\right)_{p, w_{n}} \\
& \leq \omega_{\varphi}^{r}\left(f-P_{m_{N}}^{*}, 2^{-N}\right)_{p, w_{n}}+\omega_{\varphi}^{r}\left(P_{m_{N}}^{*}, 2^{-N}\right)_{p, w_{n}} \\
& \leq c\left\|f-P_{m_{N}}^{*}\right\|_{p, w_{m_{N}}}+\omega_{\varphi}^{r}\left(P_{m_{N}}^{*}, 2^{-N}\right)_{p, w_{m_{N}}} \\
& \leq c E_{m_{N}}(f)_{p, w_{m_{N}}}+\omega_{\varphi}^{r}\left(P_{m_{N}}^{*}, 2^{-N}\right)_{p, w_{m_{N}}} .
\end{aligned}
$$

Now, the fact that $w \in \mathcal{W}_{\Lambda}^{\delta, \gamma}$ implies (see (2.6) $)$

$$
w_{m_{N}}(x) \varphi(x)^{\gamma} \leq \Lambda m_{N}^{\delta} m_{j}^{\gamma-\delta} \rho_{m_{j}}(x)^{\gamma} w_{m_{j}}(x), \quad 0 \leq j \leq N .
$$

Hence, using

$$
P_{m_{N}}^{*}=P_{1}^{*}+\sum_{j=0}^{N-1}\left(P_{m_{j+1}}^{*}-P_{m_{j}}^{*}\right)
$$

and Lemmas 7.2 and 6.1 we have

$$
\begin{aligned}
\omega_{\varphi}^{r}\left(P_{m_{N}}^{*}, 2^{-N}\right)_{p, w_{m_{N}}} & \leq \sum_{j=0}^{N-1} \omega_{\varphi}^{r}\left(P_{m_{j+1}}^{*}-P_{m_{j}}^{*}, 2^{-N}\right)_{p, w_{m_{N}}} \\
& \leq c \sum_{j=0}^{N-1} 2^{-N r}\left\|w_{m_{N}}^{1 / \lambda_{p}} \varphi^{r}\left(P_{m_{j+1}}^{*}-P_{m_{j}}^{*}\right)^{(r)}\right\|_{p} \\
& \leq c \sum_{j=0}^{N-1} 2^{-N r}\left\|m_{N}^{\delta / \lambda_{p}} m_{j}^{(\gamma-\delta) / \lambda_{p}} \rho_{m_{j}}^{\gamma / \lambda_{p}} \varphi^{r-\gamma / \lambda_{p}} w_{m_{j}}^{1 / \lambda_{p}}\left(P_{m_{j+1}}^{*}-P_{m_{j}}^{*}\right)^{(r)}\right\|_{p} .
\end{aligned}
$$

Since $r-\gamma / \lambda_{p} \geq 0$ and $\varphi \leq m_{j} \rho_{m_{j}}$, this yields 


$$
\begin{aligned}
\omega_{\varphi}^{r}\left(P_{m_{N}}^{*}, 2^{-N}\right)_{p, w_{m_{N}}} & \leq c \sum_{j=0}^{N-1} 2^{-N r}\left\|m_{N}^{\delta / \lambda_{p}} m_{j}^{r-\delta / \lambda_{p}} \rho_{m_{j}}^{r} w_{m_{j}}^{1 / \lambda_{p}}\left(P_{m_{j+1}}^{*}-P_{m_{j}}^{*}\right)^{(r)}\right\|_{p} \\
& \leq c \sum_{j=0}^{N-1} 2^{-(N-j)\left(r-\delta / \lambda_{p}\right)}\left\|\rho_{m_{j}}^{r} w_{m_{j}}^{1 / \lambda_{p}}\left(P_{m_{j+1}}^{*}-P_{m_{j}}^{*}\right)^{(r)}\right\|_{p} \\
& \leq c \sum_{j=0}^{N-1} 2^{-(N-j)\left(r-\delta / \lambda_{p}\right)}\left\|w_{m_{j}}^{1 / \lambda_{p}}\left(P_{m_{j+1}}^{*}-P_{m_{j}}^{*}\right)\right\|_{p} \\
& \leq c \sum_{j=0}^{N-1} 2^{-(N-j)\left(r-\delta / \lambda_{p}\right)} E_{m_{j}}(f)_{p, w_{m_{j}}} .
\end{aligned}
$$

Therefore,

$$
\omega_{\varphi}^{r}\left(f, n^{-1}\right)_{p, w_{n}} \leq c \sum_{j=0}^{N} 2^{-(N-j)\left(r-\delta / \lambda_{p}\right)} E_{m_{j}}(f)_{p, w_{m_{j}}}
$$

and so

$$
\begin{aligned}
\omega_{\varphi}^{r}\left(f, n^{-1}\right)_{p, w_{n}} & \leq \frac{c}{n^{r-\delta / \lambda_{p}}} \sum_{j=0}^{N} 2^{j\left(r-\delta / \lambda_{p}\right)} E_{m_{j}}(f)_{p, w_{m_{j}}} \\
& \leq \frac{c}{n^{r-\delta / \lambda_{p}}}\left(E_{1}(f)_{p, w_{1}}+\sum_{j=1}^{N} \sum_{k=m_{j-1}+1}^{m_{j}} k^{r-1-\delta / \lambda_{p}} E_{k}(f)_{p, w_{k}}\right) \\
& \leq \frac{c}{n^{r-\delta / \lambda_{p}}} \sum_{k=1}^{n} k^{r-1-\delta / \lambda_{p}} E_{k}(f)_{p, w_{k}} .
\end{aligned}
$$

We have the following immediate corollaries of Theorems 5.3 and 7.3 ,

Corollary 7.6. Let $r \in \mathbb{N}, 1 \leq p \leq \infty$ and $f \in \mathbb{L}_{p}[-1,1]$. Suppose that $w$ is a doubling weight from the class $\mathcal{W}^{\delta, \gamma}$ with $\gamma \leq r \lambda_{p}$. Then, for $0<\alpha<r-\delta / \lambda_{p}$, we have

$$
E_{n}(f,[-1,1])_{p, w_{n}}=O\left(n^{-\alpha}\right) \Longleftrightarrow \omega_{\varphi}^{r}\left(f, n^{-1}\right)_{p, w_{n}}=O\left(n^{-\alpha}\right) .
$$

Again, taking into account that any doubling weight belongs to the class $\mathcal{W}_{1}^{1,1}$ and that $1 \leq r \lambda_{p}$, for all $r \in \mathbb{N}$ and $1 \leq p \leq \infty$, we get the following corollaries (or one can obtain them as a consequence of Theorem 5.3 and Corollary [7.4).

Corollary 7.7 ( $1<p<\infty$ and all doubling weights). Let $w$ be a doubling weight, $r \in \mathbb{N}, 1<p<\infty$ and $f \in \mathbb{L}_{p}[-1,1]$. Then, for $0<\alpha<r-1 / p$, we have

$$
E_{n}(f,[-1,1])_{p, w_{n}}=O\left(n^{-\alpha}\right) \Longleftrightarrow \omega_{\varphi}^{r}\left(f, n^{-1}\right)_{p, w_{n}}=O\left(n^{-\alpha}\right) .
$$

Clearly, this corollary is also valid for $p=1$ and $p=\infty$. However, since $r-1 / \lambda_{p}=$ $r-1$ in both of these cases it seems more natural to state them in the following form replacing $r-1$ with $r$. 
Corollary 7.8 ( $p=1$ or $p=\infty$, and all doubling weights). Let $w$ be a doubling weight, $r \in \mathbb{N}, p=1$ or $p=\infty$, and $f \in \mathbb{L}_{p}[-1,1]$. Then, for $0<\alpha<r$, we have

$$
E_{n}(f,[-1,1])_{p, w_{n}}=O\left(n^{-\alpha}\right) \Longleftrightarrow \omega_{\varphi}^{r+1}\left(f, n^{-1}\right)_{p, w_{n}}=O\left(n^{-\alpha}\right) .
$$

In the case $p=\infty$, Corollary 7.8 was proved in [13] (with $\omega_{\varphi}^{r+2}$ instead of $\omega_{\varphi}^{r+1}$ ). Also, it was shown in [13, p. 183] that, in the case $r=1$ and $p=\infty$, Corollary 7.8 is no longer true if $\omega_{\varphi}^{r+1}$ is replaced by $\omega_{\varphi}^{r}$.

\subsection{Inverse theorem: the case $0<p<1$}

Theorem 7.9. Let $0<p<1, f \in \mathbb{L}_{p}[-1,1]$ and let $r \in \mathbb{N}$, and suppose that $w$ is a doubling weight from the class $\mathcal{W}_{\Lambda}^{\delta, \gamma}$ with $\gamma \leq$ rp. Then

$$
\omega_{\varphi}^{r}\left(f, \vartheta n^{-1}\right)_{p, w_{n}} \leq \frac{c}{n^{r-\delta / p}}\left(\sum_{k=1}^{n} k^{r p-\delta-1} E_{k}(f)_{p, w_{k}}^{p}\right)^{1 / p}
$$

where $\vartheta$ is the constant from Corollary [6.8, and the constant $c$ depends only on $r, p$ and the doubling constant of $w$.

We now recall that any doubling weight $w$ belongs to the class $\mathcal{W}^{\delta, \gamma}$ with $(\delta, \gamma) \in \Upsilon$. In particular, $w$ belongs to the class $\mathcal{W}^{\delta_{0}, \gamma_{0}}$ with $\gamma_{0}:=\min \{r p, 1\}$ and $\delta_{0}:=2-\gamma_{0}$. Hence, we get a corollary of Theorem 7.9 for all $r \in \mathbb{N}, 0<p<1$, and doubling weights $w$ with $\delta_{0}=2-\min \{r p, 1\}$. However, in the case $r p \leq 1$ this corollary is useless since the resulting inequality

$$
\omega_{\varphi}^{r}\left(f, \vartheta n^{-1}\right)_{p, w_{n}} \leq c n^{2(1 / p-r)}\left(\sum_{k=1}^{n} k^{2 r p-3} E_{k}(f)_{p, w_{k}}^{p}\right)^{1 / p}
$$

simply means that $\omega_{\varphi}^{r}\left(f, \vartheta n^{-1}\right)_{p, w_{n}}$ is bounded above by a quantity larger than $c E_{1}(f)_{p, w_{1}}$ which is worse than what Lemma 7.1 implies.

Therefore, we do not really get anything useful that is valid for all doubling weights if $r p \leq 1$. In the case $r p>1, \delta_{0}=\gamma_{0}=1$, and we are back to the same situation as in the case for $p \geq 1$, i.e., we can use the fact that any doubling weight is in $\mathcal{W}_{1}^{1,1}$. Hence, we get the following inverse theorem that is valid for all doubling weights.

Corollary 7.10. Let $w$ be a doubling weight, $0<p<1, f \in \mathbb{L}_{p}[-1,1]$, and let $r \in \mathbb{N}$ be such that $r>1 / p$. Then

$$
\omega_{\varphi}^{r}\left(f, \vartheta n^{-1}\right)_{p, w_{n}} \leq \frac{c}{n^{r-1 / p}}\left(\sum_{k=1}^{n} k^{r p-2} E_{k}(f)_{p, w_{k}}^{p}\right)^{1 / p}
$$

where $\vartheta$ is the constant from Corollary [6.8, and the constant $c$ depends only on $r, p$ and the doubling constant of $w$.

Remark 7.11. Since any weight that satisfies the $A^{*}$ property is in $\mathcal{W}^{0,0}$ (see Remark 2.4), it immediately follows from Theorem 7.9 that, for $A^{*}$ weights $w$, we have

$$
\omega_{\varphi}^{r}\left(f, \vartheta n^{-1}\right)_{p, w_{n}} \leq \frac{c}{n^{r}}\left(\sum_{k=1}^{n} k^{r p-1} E_{k}(f)_{p, w_{k}}^{p}\right)^{1 / p}, \quad 0<p<1 .
$$

In fact, it is possible to show that one can set $\vartheta=1$ in this case. 
Proof of Theorem 7.9. The method of the proof is rather standard (see e.g. [6]). The beginning is the same as in the case $1 \leq p \leq \infty$. Namely, let $P_{n}^{*} \in \Pi_{n}$ denote a polynomial of (near) best approximation to $f$ with weight $w_{n}$, i.e.,

$$
\left\|f-P_{n}^{*}\right\|_{p, w_{n}} \leq c E_{n}(f)_{p, w_{n}} .
$$

We let $N \in \mathbb{N}$ be such that $2^{N} \leq n<2^{N+1}$, denote $m_{j}:=2^{j}$, and recall that $\vartheta$ is the constant from Corollary 6.8.

Recalling that $w_{n}(x) \sim w_{m}(x)$ if $n \sim m$, and using Lemma 7.1 we have

$$
\begin{aligned}
\omega_{\varphi}^{r}\left(f, \vartheta n^{-1}\right)_{p, w_{n}}^{p} & \leq \omega_{\varphi}^{r}\left(f, \vartheta 2^{-N}\right)_{p, w_{n}}^{p} \\
& \leq \omega_{\varphi}^{r}\left(f-P_{m_{N}}^{*}, \vartheta 2^{-N}\right)_{p, w_{n}}^{p}+\omega_{\varphi}^{r}\left(P_{m_{N}}^{*}, \vartheta 2^{-N}\right)_{p, w_{n}}^{p} \\
& \leq c\left\|f-P_{m_{N}}^{*}\right\|_{p, w_{m_{N}}}^{p}+\omega_{\varphi}^{r}\left(P_{m_{N}}^{*}, \vartheta 2^{-N}\right)_{p, w_{m_{N}}}^{p} \\
& \leq c E_{m_{N}}(f)_{p, w_{m_{N}}}^{p}+\omega_{\varphi}^{r}\left(P_{m_{N}}^{*}, \vartheta 2^{-N}\right)_{p, w_{m_{N}}}^{p} .
\end{aligned}
$$

Using

$$
P_{m_{N}}^{*}=P_{1}^{*}+\sum_{j=0}^{N-1}\left(P_{m_{j+1}}^{*}-P_{m_{j}}^{*}\right)
$$

and Corollary 6.8 with $t:=2^{-N}$ (noting that $t \leq 1 / m_{j+1}$ for all $0 \leq j \leq N-1$ ) we have

$$
\begin{aligned}
\omega_{\varphi}^{r}\left(P_{m_{N}}^{*}, \vartheta 2^{-N}\right)_{p, w_{m_{N}}}^{p} & \leq \sum_{j=0}^{N-1} \omega_{\varphi}^{r}\left(P_{m_{j+1}}^{*}-P_{m_{j}}^{*}, \vartheta 2^{-N}\right)_{p, w_{m_{N}}}^{p} \\
& \leq \sum_{j=0}^{N-1}\left(\frac{m_{N}}{m_{j+1}}\right)^{\delta}\left(2^{-N} m_{j+1}\right)^{r p}\left\|P_{m_{j+1}}^{*}-P_{m_{j}}^{*}\right\|_{p_{w_{m_{j}+1}}}^{p} \\
& \leq c \sum_{j=0}^{N-1} 2^{-(N-j)(r p-\delta)} E_{m_{j}}(f)_{p, w_{m_{j}}}^{p} .
\end{aligned}
$$

Hence,

$$
\omega_{\varphi}^{r}\left(f, \vartheta n^{-1}\right)_{p, w_{n}}^{p} \leq c \sum_{j=0}^{N} 2^{-(N-j)(r p-\delta)} E_{m_{j}}(f)_{p, w_{m_{j}}}^{p},
$$

and so as in the proof for $1 \leq p \leq \infty$, we conclude that

$$
\begin{aligned}
\omega_{\varphi}^{r}\left(f, \vartheta n^{-1}\right)_{p, w_{n}}^{p} & \leq \frac{c}{n^{r p-\delta}} \sum_{j=0}^{N} 2^{j(r p-\delta)} E_{m_{j}}(f)_{p, w_{m_{j}}}^{p} \\
& \leq \frac{c}{n^{r p-\delta}}\left(E_{1}(f)_{p, w_{1}}^{p}+\sum_{j=1}^{N} \sum_{k=m_{j-1}+1}^{m_{j}} k^{r p-\delta-1} E_{k}(f)_{p, w_{k}}^{p}\right) \\
& \leq \frac{c}{n^{r p-\delta}} \sum_{k=1}^{n} k^{r p-\delta-1} E_{k}(f)_{p, w_{k}}^{p} .
\end{aligned}
$$

We have the following immediate corollary of Theorems 5.3 and 7.9 , 
Corollary $7.12(0<p<1)$. Let $r \in \mathbb{N}, 0<p<1$ and $f \in \mathbb{L}_{p}[-1,1]$. Suppose that $w$ is a doubling weight from the class $\mathcal{W}^{\delta, \gamma}$ with $\gamma \leq r p$. Then, for $0<\alpha<r-\delta / p$, we have

$$
E_{n}(f,[-1,1])_{p, w_{n}}=O\left(n^{-\alpha}\right) \Longleftrightarrow \omega_{\varphi}^{r}\left(f, n^{-1}\right)_{p, w_{n}}=O\left(n^{-\alpha}\right) .
$$

We remark that, since $1 \in \mathcal{W}^{0,0}$, an immediate consequence of Corollary 7.12 is the usual equivalence result for unweighted polynomial approximation in $\mathbb{L}_{p}$ for $0<p<1$.

Again, taking into account that any doubling weight belongs to the class $\mathcal{W}^{1,1}$ and assuming that $r p>1$ we get the following corollary.

Corollary $7.13(0<p<1$ and all doubling weights). Let $w$ be a doubling weight, $0<p<1, f \in \mathbb{L}_{p}[-1,1]$, and let $r \in \mathbb{N}$ be such that $r>1 / p$. Then, for $0<\alpha<r-1 / p$, we have

$$
E_{n}(f,[-1,1])_{p, w_{n}}=O\left(n^{-\alpha}\right) \Longleftrightarrow \omega_{\varphi}^{r}\left(f, n^{-1}\right)_{p, w_{n}}=O\left(n^{-\alpha}\right) .
$$

As a final remark in this section, we mention that it is still an open problem to prove or disprove if Theorems 7.3 and 7.9 are sharp.

\section{$8 \quad K$-functionals and Realization}

For $f \in \mathbb{L}_{p}, r \in \mathbb{N}$ and a weight $w$, the weighted $K$-functional is defined as follows

$$
K_{r, \varphi}(f, t)_{p, w}:=\inf _{g^{(r-1)} \in \mathrm{AC}_{\mathrm{loc}}}\left(\|f-g\|_{p, w}+t^{r}\left\|\varphi^{r} g^{(r)}\right\|_{p, w}\right)
$$

where $\mathrm{AC}_{\text {loc }}$ is the set of all locally absolutely continuous functions on $(-1,1)$. In fact, for doubling weights $w$ we are interested in a sequence of these $K$-functionals with weights $w_{n}$, and so we define several related quantities (all of which depend on $n$ ) as follows:

$$
K_{r, \varphi_{n}}(f, t)_{p, w_{n}}:=\inf _{g^{(r-1)} \in \mathrm{AC}_{\mathrm{loc}}}\left(\|f-g\|_{p, w_{n}}+t^{r}\left\|\varphi_{n}^{r} g^{(r)}\right\|_{p, w_{n}}\right),
$$

where $\varphi_{n}(x):=\varphi(x)+1 / n=n \rho_{n}(x)$,

$$
R_{r, \varphi}(f, t)_{p, w_{n}}:=\inf _{P_{n} \in \Pi_{n}}\left(\left\|f-P_{n}\right\|_{p, w_{n}}+t^{r}\left\|\varphi^{r} P_{n}^{(r)}\right\|_{p, w_{n}}\right)
$$

and

$$
R_{r, \varphi_{n}}(f, t)_{p, w_{n}}:=\inf _{P_{n} \in \Pi_{n}}\left(\left\|f-P_{n}\right\|_{p, w_{n}}+t^{r}\left\|\varphi_{n}^{r} P_{n}^{(r)}\right\|_{p, w_{n}}\right) .
$$

Note that $R_{r, \varphi}$ and $R_{r, \varphi_{n}}$ are sometimes referred to as "realizations" of appropriate $K$ functionals or "realization functionals" (see [4,5], for example).

It is clear that

$$
\begin{aligned}
& K_{r, \varphi}(f, t)_{p, w_{n}} \leq K_{r, \varphi_{n}}(f, t)_{p, w_{n}} \leq R_{r, \varphi_{n}}(f, t)_{p, w_{n}} \quad \text { and } \\
& K_{r, \varphi}(f, t)_{p, w_{n}} \leq R_{r, \varphi}(f, t)_{p, w_{n}} \leq R_{r, \varphi_{n}}(f, t)_{p, w_{n}}, \quad t>0 .
\end{aligned}
$$

It follows from Theorem 5.3 that, if $w$ is a doubling weight, $r \in \mathbb{N}, 0<p \leq \infty$, $f \in \mathbb{L}_{p}[-1,1]$, and $\mathcal{A}>0$ is any constant, then there exists $P_{n} \in \Pi_{n}$ such that

$$
\left\|f-P_{n}\right\|_{p, w_{n}}+n^{-r}\left\|\varphi_{n}^{r} P_{n}^{(r)}\right\|_{p, w_{n}} \leq c \tilde{\omega}_{\varphi}^{r}(f, \mathcal{A} / n)_{p, w_{n}} \leq c \omega_{\varphi}^{r}(f, \mathcal{A} / n)_{p, w_{n}}, \quad n \geq r,
$$


and hence, for any constant $\mathcal{B}>0$,

$$
R_{r, \varphi_{n}}(f, t)_{p, w_{n}} \leq c \tilde{\omega}_{\varphi}^{r}(f, \mathcal{A} / n)_{p, w_{n}} \leq c \omega_{\varphi}^{r}(f, \mathcal{A} / n)_{p, w_{n}}, \quad n \geq r \quad \text { and } \quad t \leq \mathcal{B} / n,
$$

where the constant $c$ depends only on $r, p, \mathcal{A}, \mathcal{B}$, and the doubling constant of $w$.

Lemmas 7.1 and 7.2 imply that, if $w$ is a doubling weight, $1 \leq p \leq \infty, f \in \mathbb{L}_{p}[-1,1]$, $\mathcal{C}>0, \mathcal{D}>0$, and $g$ is any function such that $g^{(r-1)} \in \mathrm{AC}_{\text {loc }}$ and $\left\|\varphi^{r} g^{(r)}\right\|_{p}<\infty$, then

$$
\omega_{\varphi}^{r}(f, t)_{p, w_{n}} \leq c K_{r, \varphi}(f, \mathcal{C} t)_{p, w_{n}}, \quad 0<t \leq \mathcal{D} / n
$$

where the constant $c$ depends only on $r, p, \mathcal{C}, \mathcal{D}$, and the doubling constant of $w$.

Therefore, together with (8.1), this immediately implies the following result.

Corollary 8.1. If $w$ is a doubling weight, $1 \leq p \leq \infty, f \in \mathbb{L}_{p}[-1,1]$, and $n, r \in \mathbb{N}$ are such that $n \geq r$, and $\mathcal{A} / n \leq t \leq \mathcal{B} / n$, then

$$
\begin{aligned}
\omega_{\varphi}^{r}(f, t)_{p, w_{n}} & \sim \tilde{\omega}_{\varphi}^{r}(f, t)_{p, w_{n}} \sim K_{r, \varphi}(f, t)_{p, w_{n}} \sim K_{r, \varphi_{n}}(f, t)_{p, w_{n}} \\
& \sim R_{r, \varphi}(f, t)_{p, w_{n}} \sim R_{r, \varphi_{n}}(f, t)_{p, w_{n}},
\end{aligned}
$$

where all equivalence constants depend only on $r, p, \mathcal{A}, \mathcal{B}$, and the doubling constant of $w$.

We now turn our attention to the case $0<p<1$. Things are a bit more complicated now since, as was shown in [5], the $K$-functionals are identically zero if $0<p<1$. However, we are still able to get the equivalence of the moduli and the realization functionals.

Lemmas 6.9 and 7.1 imply that, for a doubling weight $w, 0<p<1, n \in \mathbb{N}$, and some constant $\vartheta$ depending only on $r, p$, and the doubling constant of $w$,

$$
\omega_{\varphi}^{r}(f, t)_{p, w_{n}} \leq c R_{r, \varphi}(f, t)_{p, w_{n}}, \quad 0<t \leq \vartheta / n
$$

where $c$ depends only on $r, p$ and the doubling constant of $w$. For $n \geq r$, together with (8.2), this implies, for $\mathcal{A} / n \leq t \leq \vartheta / n$,

$$
\begin{aligned}
R_{r, \varphi_{n}}(f, t)_{p, w_{n}} & \leq c \tilde{\omega}_{\varphi}^{r}(f, \mathcal{A} / n)_{p, w_{n}} \leq c \tilde{\omega}_{\varphi}^{r}(f, t)_{p, w_{n}} \leq c \omega_{\varphi}^{r}(f, t)_{p, w_{n}} \\
& \leq c R_{r, \varphi}(f, t)_{p, w_{n}} \leq c R_{r, \varphi_{n}}(f, t)_{p, w_{n}} .
\end{aligned}
$$

Suppose now that $P_{n}^{*} \in \Pi_{n}$ is a polynomial of (near) best approximation to $f$ with weight $w_{n}$, i.e., $\left\|f-P_{n}^{*}\right\|_{p, w_{n}} \leq c E_{n}(f)_{p, w_{n}}$, and consider

$$
R_{r, \varphi}^{*}(f, t)_{p, w_{n}}:=\left\|f-P_{n}^{*}\right\|_{p, w_{n}}+t^{r}\left\|\varphi^{r}\left(P_{n}^{*}\right)^{(r)}\right\|_{p, w_{n}} .
$$

Then, Lemmas 6.9 and 7.1 and Theorem 5.3 imply, for $\mathcal{A} / n \leq t \leq \vartheta / n$,

$$
\begin{aligned}
R_{r, \varphi}^{*}(f, t)_{p, w_{n}} & \leq c E_{n}(f)_{p, w_{n}}+t^{r}\left\|\varphi^{r}\left(P_{n}^{*}\right)^{(r)}\right\|_{p, w_{n}} \leq c E_{n}(f)_{p, w_{n}}+c(\mathcal{A} / n)^{r}\left\|\varphi^{r}\left(P_{n}^{*}\right)^{(r)}\right\|_{p, w_{n}} \\
& \leq c E_{n}(f)_{p, w_{n}}+c \omega_{\varphi}^{r}\left(P_{n}^{*}, \mathcal{A} / n\right)_{p, w_{n}} \leq c E_{n}(f)_{p, w_{n}}+c \omega_{\varphi}^{r}(f, \mathcal{A} / n)_{p, w_{n}} \\
& \leq c \omega_{\varphi}^{r}(f, \mathcal{A} / n)_{p, w_{n}} \leq c \omega_{\varphi}^{r}(f, t)_{p, w_{n}} .
\end{aligned}
$$

Since

$$
R_{r, \varphi}(f, t)_{p, w_{n}} \leq R_{r, \varphi}^{*}(f, t)_{p, w_{n}}
$$

together with (8.3) we get the following result. 
Corollary 8.2. If $w$ is a doubling weight, $0<p<1, f \in \mathbb{L}_{p}[-1,1]$, and $n, r \in \mathbb{N}$ are such that $n \geq r$, then there exists a positive constant $\vartheta$ depending only on $r, p$ and the doubling constant of $w$, such that, for any constant $0<\mathcal{A}<\vartheta$ and $\mathcal{A} / n \leq t \leq \vartheta / n$, we have

$$
\omega_{\varphi}^{r}(f, t)_{p, w_{n}} \sim \tilde{\omega}_{\varphi}^{r}(f, t)_{p, w_{n}} \sim R_{r, \varphi}(f, t)_{p, w_{n}} \sim R_{r, \varphi_{n}}(f, t)_{p, w_{n}} \sim R_{r, \varphi}^{*}(f, t)_{p, w_{n}}
$$

where all equivalence constants depend only on $r, p, \mathcal{A}, \vartheta$, and the doubling constant of the weight $w$.

\section{References}

[1] P. Borwein and T. Erdélyi, Polynomials and polynomial inequalities, Graduate Texts in Mathematics, vol. 161, Springer-Verlag, New York, 1995.

[2] R. A. DeVore, D. Leviatan, and X. M. Yu, Polynomial approximation in $L_{p}(0<p<1)$, Constr. Approx. 8 (1992), no. 2, 187-201.

[3] R. A. DeVore and G. G. Lorentz, Constructive approximation, Grundlehren der Mathematischen Wissenschaften [Fundamental Principles of Mathematical Sciences], vol. 303, Springer-Verlag, Berlin, 1993.

[4] Z. Ditzian, Polynomial approximation and $\omega_{\phi}^{r}(f, t)$ twenty years later, Surv. Approx. Theory 3 (2007), 106-151.

[5] Z. Ditzian, V. H. Hristov, and K. G. Ivanov, Moduli of smoothness and $K$-functionals in $L_{p}$, $0<p<1$, Constr. Approx. 11 (1995), no. 1, 67-83.

[6] Z. Ditzian, D. Jiang, and D. Leviatan, Inverse theorem for best polynomial approximation in $L_{p}, 0<$ $p<1$, Proc. Amer. Math. Soc. 120 (1994), no. 1, 151-155.

[7] Z. Ditzian and V. Totik, Moduli of smoothness, Springer Series in Computational Mathematics, vol. 9, Springer-Verlag, New York, 1987.

[8] V. K. Dzyadyk, Vvedenie v teoriyu ravnomernogo priblizheniya funktsii polinomami, Izdat. "Nauka", Moscow, 1977 (Russian).

[9] T. Erdélyi, A. Máté, and P. Nevai, Inequalities for generalized nonnegative polynomials, Constr. Approx. 8 (1992), no. 2, 241-255.

[10] K. A. Kopotun, Simultaneous approximation by algebraic polynomials, Constr. Approx. 12 (1996), no. $1,67-94$.

[11] _ Pointwise and uniform estimates for convex approximation of functions by algebraic polynomials, Constr. Approx. 10 (1994), no. 2, 153-178.

[12] K. A. Kopotun, D. Leviatan, and I. A. Shevchuk, New moduli of smoothness: weighted DT moduli revisited and applied, Constr. Approx. (to appear).

[13] G. Mastroianni and V. Totik, Best approximation and moduli of smoothness for doubling weights, J. Approx. Theory 110 (2001), no. 2, 180-199.

[14] — Weighted polynomial inequalities with doubling and $A_{\infty}$ weights, Constr. Approx. 16 (2000), no. $1,37-71$.

[15] — J Jackson type inequalities for doubling weights. II, East J. Approx. 5 (1999), no. 1, 101-116.

[16] _ Jackson type inequalities for doubling and $A_{p}$ weights, Proceedings of the Third International Conference on Functional Analysis and Approximation Theory, Vol. I (Acquafredda di Maratea, 1996), 1998, pp. 83-99.

[17] P. Nevai, Bernstein's inequality in $L^{p}$ for $0<p<1$, J. Approx. Theory 27 (1979), no. 3, 239-243. 
[18] V. Operstein, A Markov-Bernstein type inequality for algebraic polynomials in $L_{p}, 0<p<1$, J. Approx. Theory 84 (1996), no. 2, 139-144.

[19] P. P. Petrushev and V. A. Popov, Rational approximation of real functions, Encyclopedia of Mathematics and its Applications, vol. 28, Cambridge University Press, Cambridge, 1987.

[20] I. A. Shevchuk, Polynomial approximation and traces of functions continuous on a segment, Naukova Dumka, Kiev, 1992 (Russian).

[21] E. M. Stein, Harmonic analysis: real-variable methods, orthogonality, and oscillatory integrals, Princeton Mathematical Series, vol. 43, Princeton University Press, Princeton, NJ, 1993. With the assistance of Timothy S. Murphy; Monographs in Harmonic Analysis, III.

[22] G. T. Tachev, A converse theorem for the best algebraic approximation in $L_{p}[-1,1](0<p<1)$, Serdica 17 (1991), no. 2-3, 161-166. 